\title{
Triggering active galactic nuclei in hierarchical galaxy formation: disk instability vs. interactions ${ }^{\star}$
}

\author{
N. Menci, M. Gatti, F. Fiore, and A. Lamastra \\ INAF - Osservatorio Astronomico di Roma, via di Frascati 33, 00040 Monte Porzio Catone, Italy \\ e-mail: menci@oa-roma.inaf.it
}

Received 15 May 2014 / Accepted 23 June 2014

\begin{abstract}
Using a state-of-the-art semi analytic model for galaxy formation, we investigated in detail the effects of black hole $(\mathrm{BH})$ accretion triggered by disk instabilities (DI) in isolated galaxies on the evolution of the AGN population. Specifically, we took on, developed, and expanded the Hopkins \& Quataert (2011, MNRAS, 411, 1027) model for the mass inflow following disk perturbations, based on a physical description of nuclear inflows and tested against aimed $N$-body simulations. We compared the evolution of AGN due to such a DI accretion mode with that arising in a scenario where galaxy interactions (IT mode) produce the sudden destabilization of large quantities of gas feeding the AGN; this constitutes the standard AGN feeding mode implemented in the earliest versions of most semi-analytic models. To study the maximal contribution of DI to the evolution of the AGN population, we extended and developed the DI model to assess the effects of changing the assumed disk surface density profile, and to obtain lower limits for the nuclear star formation rates associated to the DI accretion mode. We obtained the following results: i) For AGN with luminosity $M_{1450} \gtrsim-26$, the DI mode can provide the $\mathrm{BH}$ accretion needed to match the observed AGN luminosity functions up to $z \approx 4.5$. In such a luminosity range and redshift, it constitutes a viable candidate mechanism to fuel AGN, and can compete with the IT scenario as the main driver of cosmological evolution of the AGN population. ii) The DI scenario cannot provide the observed abundance of high-luminosity QSO with $M_{1450} \leq-26 \mathrm{AGN}$, as well as the abundance of high-redhshift $z \gtrsim 4.5$ QSO with $M_{1450} \leq-24$. As found in our earliest works, the IT scenario provides an acceptable match to the observed luminosity functions up to $z \approx 6$. iii) The dispersion of the distributions of Eddington ratio $\lambda$ for low- and intermediate-luminosity AGN (bolometric $L_{\mathrm{AGN}}=10^{43}-10^{45} \mathrm{erg} \mathrm{s}^{-1}$ ) is predicted to be much smaller in the DI scenario compared to the IT mode. iv) The above conclusions concerning the DI mode are robust with respect to the explored variants of the DI model. We discuss the physical origin of our findings. Finally, we discuss how it is possible to pin down the dominant fueling mechanism of AGN in the low-intermediate luminosity range $M_{1450} \gtrsim-26$ where the DI and the IT modes are both viable candidates as the main drivers of the AGN evolution. We show that an interesting discriminant could be provided by the fraction of AGN with high Eddington ratios $\lambda \geq 0.5$, since it increases with luminosity in the IT case, while the opposite is true in the DI scenario.
\end{abstract}

Key words. galaxies: formation - galaxies: active - galaxies: evolution - galaxies: interactions

\section{Introduction}

Understanding the processes driving the growth and evolution of the supermassive black holes (BHs) constitutes a major goal in astrophysics. At the beginning of the past decade, it became widely accepted that the such an evolution is strongly correlated with that of the host galaxies, owing to the observed tightness of the relations between the $\mathrm{BH}$ mass and the galaxy properties, such as the stellar mass or the velocity dispersion of the bulge (Kormendy \& Richstone 1995; Magorrian et al. 1998, Ferrarese \& Merritt 2000; Gebhardt et al. 2000; Marconi \& Hunt 2003; Häring \& Rix 2004; Aller \& Richstone 2007). Together with the observed low fraction of active galactic nuclei (AGN) and with the strong cosmological evolution of the number density of bright AGNs (see, e.g., Richards et al. 2006; Croom et al. 2009; Ueda et al. 2014; for a review see Merloni \& Heinz 2013), these measured relations strongly indicated that a major fraction of the $\mathrm{BH}$ mass results from accretion of gas during relatively short (duration $\tau \approx 10^{7}$ yrs) but intense (accretion rates $\dot{M}_{\mathrm{c}} \approx 1-10 M_{\odot} / \mathrm{yr}$ ), repeated bursts (see, e.g., Shankar et al. 2009; Fiore et al. 2012).

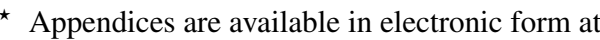
http: //www . aanda.org
}

Given this framework, the focus has progressively shifted towards understanding the triggering mechanisms responsible for such intense and short accretion episodes in the galaxy nuclei, and on their relation with the cosmological evolution of galaxies. However, connecting the nuclear accretion with the large-scale properties of galaxies is not an easy task: even the matter located at a radius of a few hundred $\mathrm{pc}$ from the central $\mathrm{BH}$ must lose more than $99.99 \%$ of its angular momentum to be effectively accreted. Numerical simulations on galactic scales have shown that gravitational torques induced by mergers (Hernquist 1989; Barnes \& Hernquist 1991; 1996; Heller \& Shlosman 1994) or instabilities in self-gravitating disks (Hernquist \& Mihos 1995; Bournaud et al. 2005; Eliche-Moral et al. 2008; Younger et al. 2008) - especially in gas rich, high-redshift galaxies (Bournaud et al. 2007; Genzel et al. 2008) - are effective in causing gas inflow down to scales $\lesssim 1 \mathrm{kpc}$. However, both theoretical arguments (Schwarz 1984; Combes \& Gerin 1985; Shlosman et al. 1989; Athanassoula 1992) and observational results (see Jogee 2006 for a review) strongly indicate that the transport of gas from galactic scales down to the pc scale (where the $\mathrm{BH}$ gravity dominates) requires additional physics.

Again, both external and internal triggers may be effective in providing additional loss of angular momentum. In the case 
of major mergers, as violent relaxation starts, the gas experiences rapidly varying gravitational torques and suffers strong shocks which can effectively result in large gas inflows in the central region (see Hopkins et al. 2010 and references therein). In the case of isolated galaxies, recent simulations show that as the disky material at smaller radii becomes self-gravitating the large-scale bars might indeed trigger a cascade of secondary gravitational instabilities that transport the gas towards the centre (Hopkins \& Quataert 2010); the latter process resembles the "bars within bar" scenario (see Shlosman et al. 1989; Friedli \& Martinet 1993; Heller \& Shlosman 1994; Maciejewski \& Sparke 2000) but includes a wider range of morphologies in the nuclear region.

Assessing the role of such processes in determining the cosmological co-evolution of AGN and galaxies is a challenging task. On the observational side, the situation is complex. On the one hand, a correlation between strong galaxy interactions and the AGN activity is found in very luminous QSOs with high mass-accretion rates $\gtrsim 10 M_{\odot} \mathrm{yr}^{-1}$ (Disney et al. 1995; Bahcall et al. 1997; Kirhakos et al. 1999; Hutchings 1987; Yates et al. 1989; Villar-Martin 2011, 2012; Treister et al. 2012). Recently, a clear statistical increase in the AGN fraction in close pairs of galaxies has been found by Ellison et al. (2011), while minor mergers as triggers for lower luminosity AGNs has been proposed to explain the observational features in some nearby galaxies (see, e.g., Combes et al. 2009). The discovery of double AGNs (e.g., Hennawi et al. 2010; McGurk et al. 2011) adds support to a merger origin of the AGN activity. On the other hand, various observational studies suggest that moderately luminous AGN are typically not major-merger-driven at $z \lesssim 1$ (Salucci et al. 1999; Grogin et al. 2005; Pierce et al. 2007; Georgakakis et al. 2009; Cisternas et al. 2011; Villforth et al. 2014), and interestingly also at $z \approx 2$ (Rosario et al. 2013a; Silverman et al. 2011; Kocevski et al. 2012). Bournaud et al. (2012) find a correlation between the presence of violent instabilities and AGN activity in gas-rich galaxies at high redshift, thanks to the presence of giant clumps of gas in the disk.

On the theoretical side, aimed, high-resolution $N$-body simulations can study specific galaxy systems, but understanding the relative effects of the disk instability (DI) and of the galaxy interactions (IT thereafter) as AGN feeding modes requires implementing the physics of nuclear gas inflows into cosmological models of galaxy formation. In turn, this requires an analytical description of such processes to be implemented into existing semi-analytic models (see Baugh 2006 for an introduction) or as sub-grid physics in cosmological simulations. As for the IT scenario, multi-scale high-resolution hydrodynamical $N$-body simulations are now resolving scales $\sim 10 \mathrm{pc}$, showing that collisions efficiently cause most of the gas to flow to the centre on timescales of a few dynamical times (see Hopkins et al. 2010 and references therein); analytical - though simplified - or phenomenological descriptions of the inflow determined by mergers have been developed by several authors (see, e.g., Makino \& Hut 1997; Cavaliere \& Vittorini 2000) and then calibrated and tested against binary hydrodynamic merger simulations (Robertson et al. 2006a,b; Cox et al. 2006; Hopkins et al. 2007b); here the fraction of galactic gas which is able to lose angular momentum and flow to the centre is related to large relative variations $\Delta j / j$ of the gas specific angular momentum induced by the interactions, which is in turn connected to the mass ratio $M^{\prime} / M$ of the merging partners.

A different situation holds for the DI scenario. Until recently, no analytic theory that accurately predicts the inflow rates of gas in mixed stellar-plus-gas systems has been worked out; most of the existing works have been based on the over-simplified assumption of completely stellar or gaseous disks (see, e.g., Goldreich \& Lynden-Bell 1965; Lin et al. 1969; Toomre 1969; Lynden-Bell \& Kalnajs 1972; Goldreich \& Tremaine 1980; Binney \& Tremaine 1987; Shu et al. 1990; Ostriker et al. 1992). In fact, the inflows driven by disk instability have been so far implemented into semi-analytic models only through ansatz concerning the fate of disk gas and stars when the disk becomes unstable; for example, the model by Hirshman et al. (2012) assumes that a tunable fraction of the gas mass exceeding the threshold for instability is accreted onto the $\mathrm{BH}$, while Fanidakis et al. (2012) assume that all gas and stars in the disk are transferred to the bulge component, leading to more dramatic effects. Owing to the lack of an analytical description of the complex physics involved in the transport of gas in the nuclear region, the results strongly depend on the assumed prescriptions and may actually over-estimate the real inflows attainable in the central regions under realistic conditions.

Recently, however, a step forward in the description of the gas inflow due to disk instabilities has been proposed by Hopkins \& Quataert (2011; HQ11 hereafter). The authors develop an analytic model that attempts to account for the physics of angular momentum transport and gas inflow from galactic scales $(\approx 1 \mathrm{kpc})$ inwards to the nuclear scales $\sim 10 \mathrm{pc}$ where the $\mathrm{BH}$ potential is dominant. The multi-component nature of the disk is considered, and the inflow towards the $\mathrm{BH}$ is related to the nuclear star formation. Such a model was tested by the authors against pointed hydrodynamical simulations, so that at present it constitutes a solid baseline for describing the $\mathrm{BH}$ accretion due to disk instabilities.

In this work, we include the HQ11 model for disk instability in an updated version of the Rome semi-analytic model of galaxy formation (Menci et al. 2006, 2008) to compute the statistical effects of such an accretion mode on the AGN population, within a cosmological context. The results are compared with those obtained by assuming interactions as the only trigger of AGN activity (our reference model). It is important to emphasize that here we do not attempt to develop a best-fitting model, tuning the relative role of the two accretion triggers. Our aim is to study the maximal effects of a physical model for $\mathrm{BH}$ accretion triggered by disk instabilities onto the AGN and BH statistics and to enlighten the main differences with respect to the predictions of purely interaction-driven models for AGN feeding. The final goal - after comparing with observations - is to single out the physical regimes where the different feeding modes may be effective.

The paper is organized as follows. In Sect. 3 we recall the main properties of the semi-analytic model that we use, describing the modifications with respect to the previous version, and test the updated model by comparing the predicted evolution of the galaxy population with existing observations. In Sect. 4 we describe how we implement different $\mathrm{BH}$ accretion mechanisms into our semi-analytic model. In Sect. 4.1 we recall our reference model based on galaxy interactions, while in Sect. 4.2 we present our implementation of the HQ11 model for accretion from disk instabilities. We also present extensions of the HQ11 model obtained by relaxing or modifying some of the assumptions of the original HQ11 model. We present our results in Sect. 5, and Sect. 6 is devoted to discussion and conclusions.

\section{The semi-analytic model}

Our work is based on the semi-analytic model described in Menci et al. (2006, 2008), to which we refer for details. Here we 
recall its key points and describe the main updates. The backbone is constituted of the merging trees of dark matter haloes, generated through a Monte Carlo procedure on the basis of the merging rates given by the Extended Press \& Schechter (EPS) formalism (see Bardeen et al. 1991; Lacey \& Cole 1993; Bower 1991). After each merging event, the dark matter haloes included in a larger object may survive as satellites, or sink to the centre owing to dynamical friction to increase the mass of the central dominant galaxy. The baryonic processes taking place in each dark matter halo are then computed. The gas in the halo, initially set to have a density given by the universal baryon fraction and to be at the virial temperature, cools due to atomic processes and settles into a rotationally supported disk with mass $M_{\mathrm{c}}$, disk radius $R_{\mathrm{d}}$, and disk circular velocity $V_{\mathrm{d}}$, computed as in Mo et al. (1998). The cooled gas $M_{\mathrm{c}}$ is gradually converted into stars, with a rate $\dot{M}_{*}=M_{\mathrm{c}} / \tau_{*}$ given by the Schmidt-Kennicut law with $\tau_{*}=1$ Gyr. In addition to the above "quiescent" mode of star formation, galaxy interactions occurring in the same host dark matter halo may induce the sudden conversion of a fraction $f$ of cold gas into stars on a short time-scale $\sim 10^{7}-10^{8}$ yrs given by the duration of the interaction. The fraction $f$ is related to the mass ratio and to the relative velocity of the merging partners as described in Menci et al. (2003). The energy released by the supernovae associated to the total star formation returns a fraction of the disk gas into the hot phase, providing the feedback needed to prevent over-cooling. An additional source of feedback is provided by the energy radiated by the AGN, which corresponds to the active accretion phase of the central $\mathrm{BH}$; the detailed description of our implementation of the AGN feedback is given in Menci et al. (2008). In our reference model, the triggering and the luminosity of AGN are determined by galaxy interactions as described in Sect. 4.1. Finally, the luminosity - in different bands - produced by the stellar populations of the galaxies are computed by convolving the star formation histories of the galaxy progenitors with a synthetic spectral energy distribution, which we take from Bruzual \& Charlot (2003) assuming a Salpeter initial mass function.

With respect to the model version described in our previous works, we performed some changes that we describe below. First, we included a treatment of the transfer of stellar mass to the bulge during mergers. A canonical prescription in semianalytic models (see Khochfar \& Silk 2006; Parry Eke \& Frenk 2009; Benson \& Devereux 2010; De Lucia et al. 2011) is to assume that the stellar content of galaxies is transferred to the bulge during major mergers characterized by stellar mass ratios $\mu=M_{* 1} / M_{* 2}$ of the merging partners that are larger than some fixed fraction $0.2-0.3$ calibrated through $N$-body simulations. Recently, Hopkins et al. (2009) have shown that the distribution of the bulge-to-total $(\mathrm{B} / \mathrm{T})$ ratio provides a better match to the observations when the transferred stellar mass in mergers is a function of both merger mass ratio and gas fraction in the progenitor galaxies. Following these authors, we assume that in mergers with $\mu \geq 0.2$ only a fraction $1-f_{\text {gas }}$ of the disk mass is transferred to the bulge. The resulting $B / T$ distribution is compared with observations from Fisher \& Drory (2011) and Lackner \& Gunn (2012) in Fig. 1; however, our next results (Sect. 5) will not change appreciably if we make the canonical assumption that all disk stars are transferred into the bulge during major mergers.

A second modification of our original model was to introduce a description of the tidal stripping of stellar material from satellite galaxies. We adopted exactly the treatment introduced by Henriques \& Thomas (2010) in the Munich semi-analytic model, to which we refer for details. Here we just recall that the model is based on the computation of the tidal radius for each satellite galaxy, identified as the distance from the satellite centre at which the radial forces acting on it cancel out (King 1962; Binney \& Tremaine 1987; see also Taylor \& Babul 2001). These forces are the gravitational binding force of the satellite, the tidal force from the central halo, and the centrifugal force. In the simple approximation of nearly circular orbits and of an isothermal halo density profile, such a radius can be expressed as $r_{\mathrm{t}} \approx \sigma_{\text {sat }} r_{\text {sat }} / \sqrt{2} \sigma_{\text {halo }}$, where $\sigma_{\text {sat }}$ and $\sigma_{\text {halo }}$ are the velocity dispersions of the satellite and of the halo, respectively, and $r_{\text {sat }}$ is the halocentric radius of the satellite, which we computed in our Monte Carlo code during its orbital decay to the centre due to dynamical friction. For each satellite galaxy, the material outside this radius is assumed to be disrupted and becomes a diffuse stellar component in the host halo (see Henriques \& Thomas 2010).

The above model constitutes our baseline for connecting the growth of BHs to the properties of their host galaxies. Thus, we first tested the updated version of our model to ensure it provides a reasonable account of the galactic properties and of their evolution over a wide range of cosmic times, assuming a concordance cosmology (see Spergel et al. 2007). In Fig. 1 we show how the model predictions compare with different local observables: the stellar mass function, the $B$-band luminosity function, and the ratio of the bulge to the total stellar mass. The good matching indicates that the model provides a fair description of both the (roughly) instantaneous and integrated star formation rates and that our treatment of stellar mass stripping and morphological transformations is consistent with observations.

As a further test we show in Fig. 2 (left panel) the predicted local colour-magnitude distribution, which seems to capture the well known bimodal nature of the observed distribution. To quantify such a result, we compared in detail the predicted colour distribution of galaxies with different magnitudes (the right panel) with the SDSS (Sloan Digital Sky Survey) data. Besides the mentioned colour bimodality, the fraction of red galaxies continuously increases with increasing galaxy magnitude, as indicated by the data.

Finally, we tested that the evolution of the distribution of the $K$-band luminosity (a proxy for the stellar mass content) and of the UV luminosity (a proxy for the instantaneous star formation rate) are in reasonable agreement with the observations over the whole range of cosmic times where observations are available. This is done in detail in Fig. 3, where (in the left panel) we compare our results with the observed evolution of the $K$-band luminosity function from Cirasuolo et al. (2010) up to redshifts $\approx 3$, and (in the right panel ) with the observed evolution of the UV luminosity functions of "dropout" galaxies from different authors (see caption) up to redshifts $z \approx 8$. Since the latter depends strongly on the colour selection adopted to select the galaxy sample, we performed the same cuts in the colourcolour plane as adopted in the observations to select $U$-dropout galaxies at $z \approx 3$ (Giavalisco et al. 2004) $B$-, $V$-, and $I$-dropouts (at $z \approx 4, z \approx 5, z \approx 6$, respectively, see Bouwens et al. 2007), and infra-red dropouts at $z \geq 7$ (Bouwens et al. 2011). To account for uncertainties related to adopted dust-extinction curve, our predictions are shown as shaded regions (see caption).

The comparisons above show that our semi-analytic model provides a reasonable statistical description of basic physical quantities (such as star formation rates, assembled stellar mass) that determine the cosmological evolution of the galaxy populations over a wide interval of cosmic times, so that it constitutes a solid "backbone" to connect the BH accretion (hence the ensuing AGN evolution) to the physical properties of their host galaxies, as we describe next. 

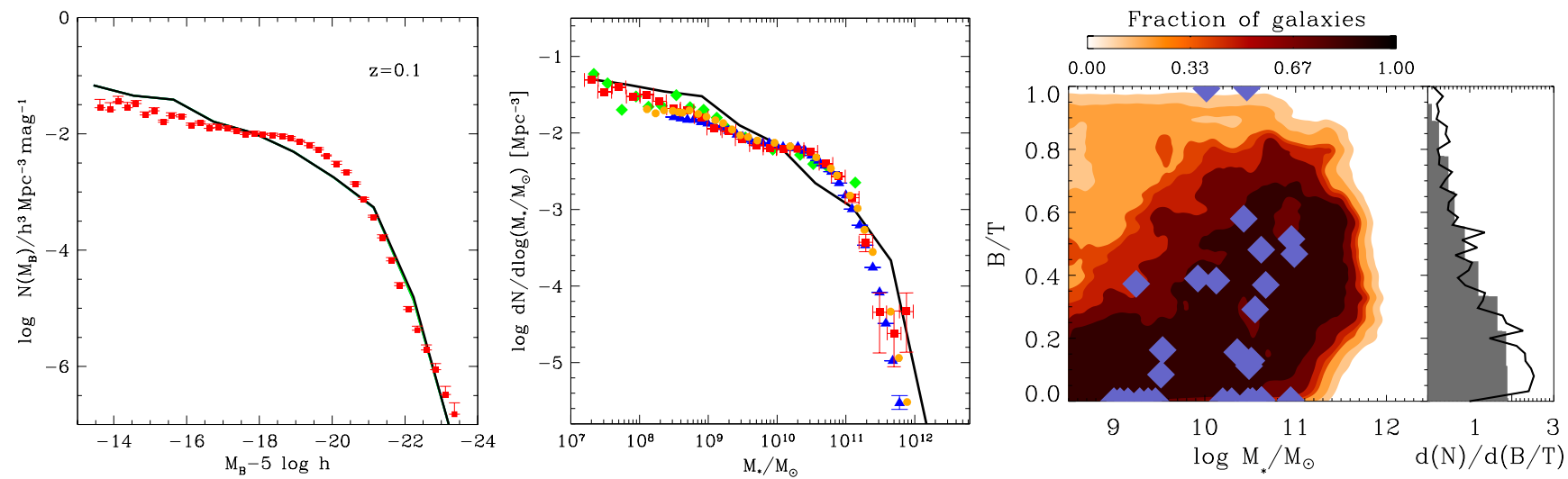

Fig. 1. Left panel: predicted luminosity function at $z \leq 0.1$ (in $b j$ band, solid line) compared with observations from Jones et al. (2006). Central panel: predicted local stellar mass function (solid line) compared with data from Baldry et al. (2012, squares), Li \& White (2009, triangles), Panter et al. (2007, circles), and Fontana et al. (2006, diamonds). Right panel: the contour plot shows the predicted relative fraction of bulge-to-total mass as a function of the galaxy stellar mass. This is compared with data from Fisher \& Drory (2011). The corresponding distribution of B/T ratios (differential number of objects per B/T bin, normalized to the total number, solid line) is also compared with the SDSS data (Lackner \& Gunn 2012, shaded histogram) in the rightmost panel, for galaxies in the magnitude range $-24 \leq M_{r} \leq-17$.

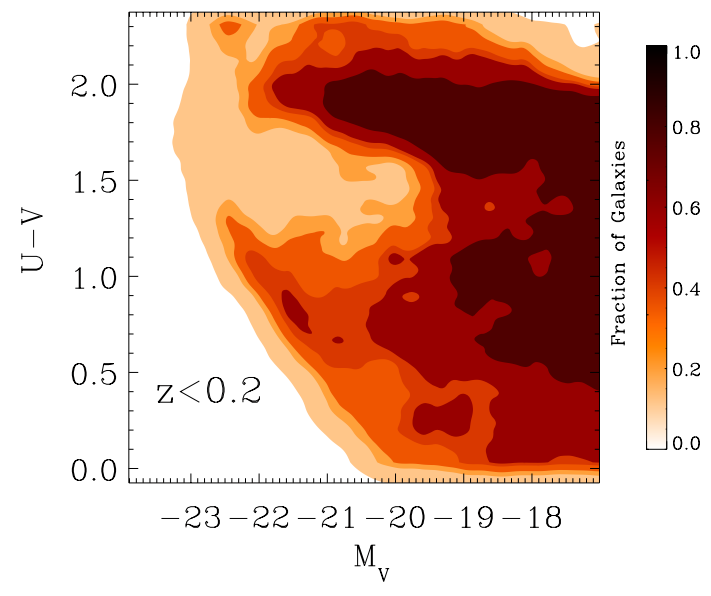

\section{Implementing the AGN feeding processes}

We have updated our semi-analytic model to include two different modes for the $\mathrm{BH}$ accretion: i) an interaction-triggered (IT) mode, where the triggering of the AGN activity is external and provided by galaxy interactions (including both mergers and flyby), which can provide - for major interactions - a large relative variation $\Delta j / j$ of the angular momentum of the galactic gas; ii) a mode where accretion occurs due to disk instabilities, where the trigger is internal and provided by the break of the axial symmetry in the distribution of the galactic cold gas. In both cases, a nuclear star formation is associated to the accretion flow triggering the AGN.

Our modelling for the IT mode has been described in previous papers (starting from Menci et al. 2004, 2008; see also Lamastra et al. 2013b), so we only give a brief account of it, to recall its main properties. In this paper we expand on our implementation of the DI mode, which constitutes a novel component of the semi-analytic model. It is based on the HQ11 analytical model. Its detailed testing against high-resolution hydrodynamical $N$-body simulations (see HQ11; Anglés-Alcázar et al. 2013) makes it a reliable baseline for describing the accretion flow onto the $\mathrm{BH}$ within the limits of the present modelling of instabilities. We recompute the HQ11 model and extend it beyond the assumption originally adopted by HQ11 to assess its robustness and to compute the maximal impact that the DI mode can have on the evolution of the AGN population under different hypotheses.

\subsection{The interaction-driven model}

As implemented in other semi-analytic models, galactic clumps within a host halo undergo different processes. First, they lose angular momentum from the dynamical friction and eventually merge with the central galaxy; such a process is implemented in our model by adopting the standard Chandrasekar expression for the dynamical friction timescale given in Lacey \& Cole (1993) that is adopted in most semi-analytic models (see also Somerville \& Primack 1999; Cole et al. 2000; for variants of such a description see Somerville et al. 2012 and references therein).

In addition, satellite galaxies with any given circular velocity $v_{\mathrm{c}}$ (and tidal radius $r_{\mathrm{t}}$ ) may interact inside a host halo with circular velocity $V$ at a rate (Menci et al. 2002)

$\tau_{r}^{-1}=n_{T}(V) \Sigma\left(r_{\mathrm{t}}, v_{\mathrm{c}}, V\right) V_{\text {rel }}(V)$,

where $n_{T}$ is the number density of galaxies in the host halo, $V_{\text {rel }}$ the relative velocity between galaxies, and $\Sigma$ the cross section for such encounters. The above expression yields the probability for fly-by encounters (not leading to a merging) for a geometrical cross section $\Sigma \sim \pi\left\langle r_{\mathrm{t}}^{2}+r_{\mathrm{t}}^{\prime 2}\right\rangle$, while it determines the probability of a merging (binary aggregation) when the geometrical cross 
N. Menci et al.: Triggering active galactic nuclei in hierarchical galaxy formation: disk instability vs. interactions
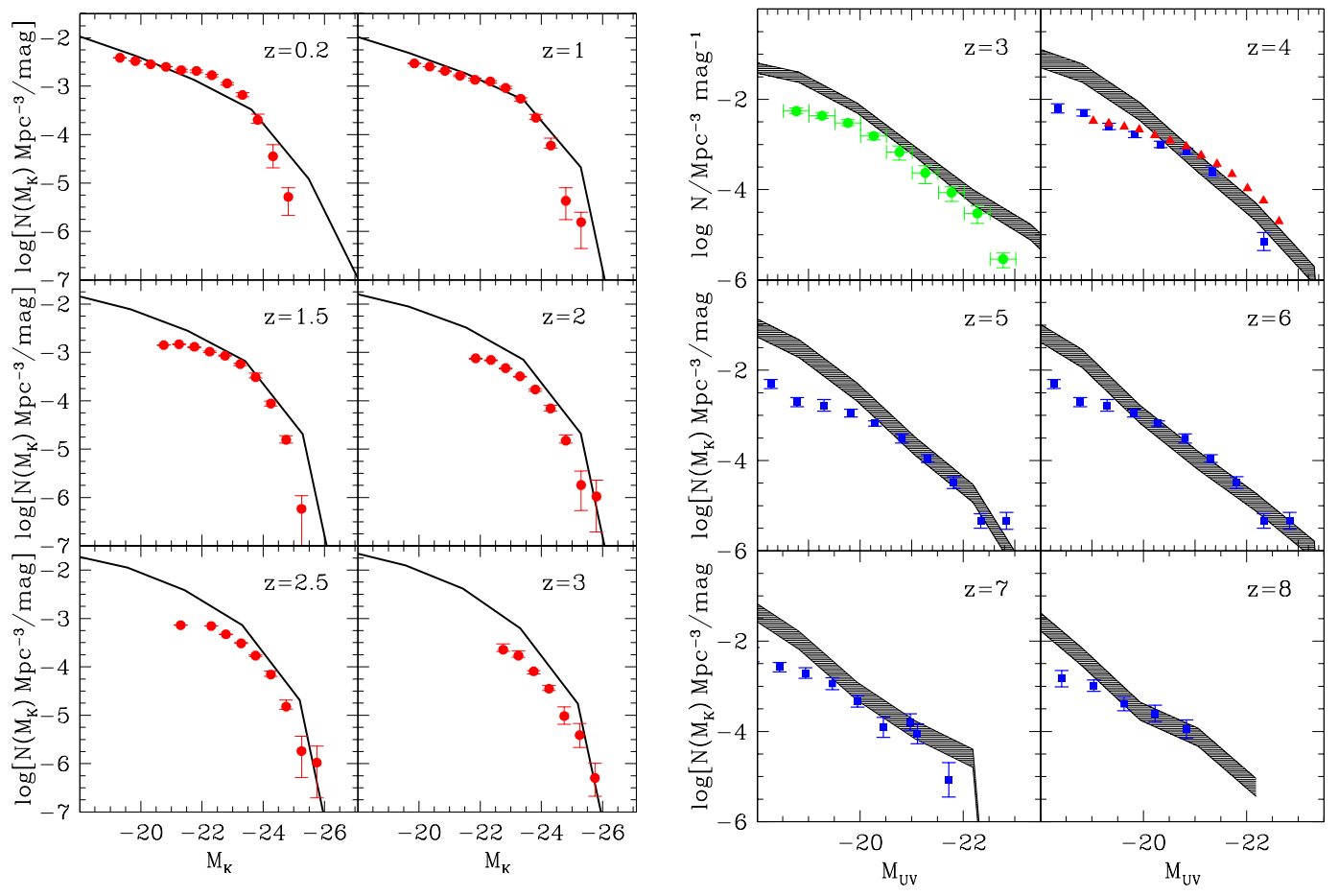

Fig. 3. Left panels: evolution of the $K$-band luminosity function predicted by the model (solid line) compared with the data from Cirasuolo et al. (2010, squares). Right panels: predicted evolution of the UV luminosity function (shaded region) compared with observed distribution of dropout galaxies. The shaded region encompasses the uncertainties due to the adopted extinction curve (Small Magellanic Cloud, Milky Way or Calzetti) In each redshift bin, the model galaxies have been selected according to the same selection criterium as adopted in the observations (see text). Data are taken from Reddy \& Steidel (2009, dots), Bouwens et al. (2007, 2011, squares), and van der Burg et al. (2010, triangles).

section is suppressed by a factor $\sim v_{\mathrm{c}}^{2} / V_{\text {rel }}^{2}$, which accounts for the requirement that the orbital energy is transferred into internal degrees of freedom. The above appropriate cross sections are given by Saslaw (1985) in terms of the tidal radius $r_{\mathrm{t}}$ associated to a galaxy with given circular velocity $v_{\mathrm{c}}$ (Menci et al. 2004). The binary merging of satellite galaxies is also implemented in other semi-analytic models (see, e.g., Somerville \& Primack 1999), and the tidal stripping of satellite galaxies is also included in our model as described in Menci et al. (2012).

The fraction $f$ of cold gas destabilized by any kind of merging or interaction has been worked out by Cavaliere \& Vittorini (2000) in terms of the variation $\Delta j$ of the specific angular momentum $j \approx G M / V_{\mathrm{d}}$ of the gas to read (Menci et al. 2004) as

$f \approx \frac{1}{2}\left|\frac{\Delta j}{j}\right|=\frac{1}{2}\left\langle\frac{M^{\prime}}{M} \frac{R_{\mathrm{d}}}{b} \frac{V_{\mathrm{d}}}{V_{\mathrm{rel}}}\right\rangle$,

where $b$ is the impact parameter, evaluated as the greater of the radius $R_{\mathrm{d}}$ and the average distance of the galaxies in the halo, $M^{\prime}$ is the mass of the partner galaxy in the interaction, and the average is performed over the probability of finding such a galaxy in the same halo where the galaxy with mass $M$ is located. The pre-factor accounts for the probability $1 / 2$ of inflow rather than outflow related to the sign of $\Delta j$. The AGN and starburst events are triggered by all galaxy interactions including major mergers $\left(M \simeq M^{\prime}\right)$, minor mergers $\left(M \ll M^{\prime}\right)$, and fly-by events. We assume that in each interaction, one fourth of the destabilized fraction $f$ feeds the central $\mathrm{BH}$, while the remaining fraction feeds the circumnuclear starbursts (Sanders \& Mirabel 1996). Thus, the $\mathrm{BH}$ accretion rate is given by

$\frac{\mathrm{d} M_{\mathrm{BH}}}{\mathrm{d} t}=\frac{1}{4} \frac{f M_{\mathrm{c}}}{\tau_{b}}$ where the time-scale $\tau_{b}=R_{\mathrm{d}} / V_{\mathrm{d}}$ is assumed to be the crossing time of the destabilized galactic disk. The fraction of destabilized gas in Eq. (2) is proportional to the mass ratio $M^{\prime} / M$ of the merging partners; thus for grazing, low speed encounters $\left(R_{\mathrm{d}} \approx b\right.$ and $V_{\text {rel }} \approx V_{\mathrm{d}}$ ) major mergers (with $M^{\prime} / M \approx 1$ ) may lead to a large relative variation in the gas angular momentum $\Delta j \approx j$ and to a high accreted fraction $f \sim 0.5$.

\subsection{The disk instability model}

In this section, we a) recall the key points in the HQ11 computation of the inflow rate onto the $\mathrm{BH}$, performed for a power-law gas density profile; b) extend such a computation to treat different (exponential) gas density profiles; c) based on the HQ11 scenario we work out the star formation rate associated to a given $\mathrm{BH}$ accretion rate; and d) discuss how the computed accretion and star formation rates from disk instabilities actually represent upper limits to the real rates. In fact, in this work we are interested in investigating the maximal contribution that - according to present modelling - DI can give to the cosmological evolution of AGN, in order to constrain the evolution physical conditions where they may compete with, or even dominate, the contribution from galaxy interactions.

\subsubsection{Computing the black hole accretion rate}

As recalled in the Introduction, gas rich mergers and tidal interactions are not the only ways to produce a net mass inflow into the central region of the galaxies. In certain cases, secular growth processes such as disk instabilities (DI) might play a crucial role in feeding the central SMBH. 
From a modelling point of view, describing such an accretion mode requires i) modelling the DI trigger; and ii) modelling the corresponding inflow rate onto the $\mathrm{BH}$. As for the first, a widely used criterion for the onset of disk instabilities is that proposed by Efstathiou et al. (1982) based on $N$-body simulations, which states that a disk becomes unstable if its mass exceeds a critical value:

$M_{\text {crit }}=\frac{v_{\max }^{2} R_{\mathrm{d}}}{G \epsilon}$,

where $R_{\mathrm{d}}$ the scale length of the disk, and $v_{\max }$ is the maximum circular velocity considering all the galaxy components. The latter is derived from velocity profiles computed assuming a Navarro Frenk \& White (1997, NFW) dark matter density profile, and it includes the effect of baryons following Mo et al. (1998), as described in Menci et al. (2005). Here $\epsilon \sim 0.5-0.75$ is a parameter calibrated on simulations. The above criterion is the one usually adopted in semi-analytic models: Hirschmann et al. (2012) adopt a value $\epsilon=0.75$, and a similar value is adopted by Fanidakis et al. (2011). ITo investigate the maximum effect of DI on the statistical evolution of AGN we also adopt the value $\epsilon=0.75$.

Computing the mass inflow rate onto the $\mathrm{BH}$, and the associated nuclear star bursts, is a more complex situation. At present, semi-analytical models only resort to ansatz tuned to maximize the model agreement with observations; in Hirschmann et al. (2012) the mass accretion rate is then calculated considering all the mass exceeding the threshold to be added in the central bulge and a tiny fraction of it $\left(\sim 10^{-3}\right.$, motivated by the local $\mathrm{BH}$-bulge mass relation) to be accreted in the central $\mathrm{BH}$ according to an exponential growth characterized by the Salpeter timescale. In the GALFORM model, Bower et al. (2006) and Fanidakis et al. (2012) assume that all the mass of the disk (not only the mass in excess) is added to the central bulge, leading to an enhanced effectiveness of the whole process. The lack of an analytical description of the complex physics processes acting in the transport of gas in the nuclear region means that the results strongly depend on the assumed prescriptions and may actually overestimate the actual inflows attainable in the central regions under realistic conditions.

Here we attempt to adopt a more physical treatment of the mass inflow from disk instabilities. In particular, we take on the analytical model proposed by HQ11, which aims at accounting for the physics of angular momentum transport and gas inflow from galactic scales $(\approx 1 \mathrm{kpc})$ inwards where the $\mathrm{BH}$ potential is dominant. It considers the multi-component nature of the disk, and the inflow towards the $\mathrm{BH}$ is related to the nuclear star formation. In addition, such a model was tested by the authors against aimed hydrodynamical simulations, so that at present it constitutes a solid base for describing the $\mathrm{BH}$ accretion due to disk instabilities.

The model investigates the response of the disk to an external non-axisymmetric perturbation of the potential $\Phi_{a}=|a| \Phi_{0}$, where the perturbation amplitude is $|a|<1$. The unperturbed system corresponds to an axisymmetric thin disk with a spherical component (BH, bulge and dark matter halo). While for periodic particle orbits the net angular momentum loss vanishes at the first order in $a$, HQ11 show that the shocks generated by gas particle in the condition of orbit crossing lead to a first-order effect. As a result, in the orbit crossing (non-linear) regime, they obtain the orbit-averaged gas inflow rate

$\frac{\mathrm{d} M_{\text {infl }}}{\mathrm{d} t}=\Sigma_{\mathrm{g}}(R) R^{2} \frac{\Omega}{2} \frac{\Phi_{a}}{V_{\mathrm{c}}^{2}} \frac{m}{1+\partial \ln V_{\mathrm{c}} / \partial \ln R}$ where $\Sigma_{\mathrm{g}}(R)$ is the gas surface density profile; $\Omega$ the angular velocity of the unperturbed disk, induced by both the bulge and the disk mass enclosed within the radius $R ; V_{\mathrm{c}}$ is the corresponding circular velocity; $m$ is the mode of the perturbations (the perturbed potential is $m$-fold symmetric, so that $m=0$ corresponds to axial symmetry); and marginal orbit crossing is assumed following HQ11. Here, our strategy is to maximize the effects of DI to single out the wider range of physical conditions where such a process may be effective in fueling AGNs. We therefore assume that when disk instabilities are triggered Eq. (5) always holds.

The mass inflow rate in Eq. (5) is of first order in perturbation amplitude, since it depends linearly on $\Phi_{a}$. To calculate the exact value of the mass inflow rate, we need the analytic expression for $\Phi_{a}$ and $\Sigma_{\mathrm{g}}$. The former is obtained in the WKB approximation where the disk dominates the potential, while it is related to the $\mathrm{BH}$ mass in the inner region where the $\mathrm{BH}$ gravity is dominant (see Appendix A). The latter can be estimated assuming an equilibrium between mass inflow rate due to the disk instability and star formation rate (described in terms of its surface density $\Sigma_{*}$ ):

$$
\frac{\partial}{\partial R} \frac{\mathrm{d} M_{\mathrm{infl}}}{\mathrm{d} t} \simeq 2 \pi R \frac{\mathrm{d} \Sigma_{*}}{\mathrm{~d} t}=2 \pi R \frac{\Sigma_{\mathrm{k}}}{t_{\mathrm{k}}}\left(\frac{\Sigma_{\mathrm{g}}}{\Sigma_{\mathrm{k}}}\right)^{\eta_{\mathrm{k}}} .
$$

The last equality follows after relating the star formation surface density $\Sigma_{*}$ to the gas surface density $\Sigma_{\mathrm{g}}$ through the Schmidt-Kennicut law $\mathrm{d} \Sigma_{*} / \mathrm{d} t=\left(\Sigma_{\mathrm{k}} / t_{\mathrm{k}}\right) *\left(\Sigma_{\mathrm{g}} / \Sigma_{\mathrm{k}}\right)^{\eta_{\mathrm{k}}}$, where the timescale $t_{\mathrm{k}}$, the normalization $\Sigma_{\mathrm{k}}$ and the exponent $\eta_{\mathrm{k}}$ are derived from fits to the observations: typical values (Bouché et al. $2007)$ are $t_{\mathrm{k}} \approx 0.4 \times 10^{9} \mathrm{yr}, \Sigma_{\mathrm{k}} \approx 10^{8} M_{\odot} \mathrm{kpc}^{-2}$, and $\eta_{\mathrm{k}}=1.7$, but the real slope $\eta_{\mathrm{k}}$ is still subject to different observational estimates in the range $1.2 \lesssim \eta_{\mathrm{k}} \lesssim 1.8$ (see, e.g., Davies et al. 2007; Hicks et al. 2009; Daddi et al. 2010; Genzel et al. 2010; Santini et al. 2014), also depending on the redshift and on the properties of the adopted galaxy sample.

There could be situations where the equilibrium described by Eq. (6) does not hold: when star formation inhibits the mass inflow completely, or when the gas inflow rate is higher than star formation (Barnes \& Hernquist, 1991). However, we later show that these two different regimes yield a mass inflow onto the central BH weaker than inflow obtained when the equilibrium is reached. Since our aim is to study the maximum effects of DI on the cosmological evolution of AGN, we retain the above approach.

With Eqs. (5) and (6) it is possible to solve for both the gas density (hence for the star formation) and the mass inflow. To this aim (see Appendix A for a step-by-step computation), the potential (hence $\Phi_{a}$ in Eq. (5)) has to be evaluated in two regions: an outer region where the disk gravity is dominant, and an inner region where gravitational potential is dominated by the $\mathrm{BH}$. The radius $R_{\mathrm{BH}} \sim 10-100 \mathrm{pc}$ (depending on the $\mathrm{BH}$ mass) marks the separations between the two regions, while the scale $R_{\text {acc }} \sim 10^{-2}-10^{-1} \mathrm{pc}$ corresponds to the inner bound of the innermost regions; inside this radius, star formation ceases and the mass inflow can be assumed to be almost constant. The mass inflow at this radius $\dot{M}_{\text {infl }}\left(R_{\text {acc }}\right)$ corresponds to the final $\mathrm{BH}$ accretion rate that we include in our semi-analytic model. The computation of such a quantity is given in detail in Appendix A, where it is expressed in terms of quantities evaluated far from the very central region, at a "galactic" scale $R_{0} \sim 100-1000 \mathrm{pc}$, so that the central $\mathrm{BH}$ accretion rate can be easily connected to the galactic properties of the host galaxy computed in our semianalytic model. This is done in Appendix A for a power-law scaling of the disk surface density $\Sigma_{\mathrm{d}} \sim R^{-\eta_{\mathrm{d}}}$ and in Appendix B for an exponential disk profile. In the first case, $\eta_{\mathrm{d}}$ is calibrated on 
$N$-body simulations: our fiducial choice is to take the best-fitting value $\eta_{\mathrm{d}}=1 / 2$ given by HQ11. Then, for a Kennicut-Schmidt index $\eta_{\mathrm{k}}=7 / 4$ (as adopted by HQ11) the final result is

$$
\begin{aligned}
\frac{\mathrm{d} M_{\mathrm{BH}}}{\mathrm{d} t} \equiv & \frac{\mathrm{d} M_{\mathrm{infl}}\left(R_{\mathrm{acc}}\right)}{\mathrm{d} t} \approx \frac{\alpha\left(\eta_{\mathrm{k}}, \eta_{\mathrm{d}}\right) f_{\mathrm{d}}^{4 / 3}}{1+2.5 f_{\mathrm{d}}^{-4 / 3}\left(1+f_{0} / f_{\mathrm{gas}}\right)}\left(\frac{M_{\mathrm{BH}}}{10^{8} M_{\odot}}\right)^{1 / 6} \\
& \times\left(\frac{M_{\mathrm{d}}\left(R_{0}\right)}{10^{9} M_{\odot}}\right)\left(\frac{R_{0}}{100 \mathrm{pc}}\right)^{-3 / 2}\left(\frac{R_{\mathrm{acc}}}{10^{-2} R_{\mathrm{BH}}}\right)^{5 / 6} M_{\odot} \mathrm{yr}^{-1}
\end{aligned}
$$

Here $M_{\mathrm{BH}}$ is the central $\mathrm{BH}$ mass, $f_{\mathrm{d}}$ is the total disk mass fraction, and $M_{\mathrm{d}}$ and $M_{\text {gas }}$ are the disk and the gas mass calculated in $R_{0}$. For physical values of $0 \leq f_{\mathrm{d}} \lesssim 0.5$, the above equation yields accretion rates that are indistinguishable from the original formulation given in HQ11. The above equation relates the $\mathrm{BH}$ accretion at the sub-resolution scale $R_{\mathrm{acc}}$ to quantities evaluated at the galactic scale $R_{0}$ which are computed for each galaxy by the semi-analytic model described in Sect. 2 . Under the assumptions adopted by HQ11 - the product $M_{\mathrm{d}}\left(R_{0}\right) R_{0}^{-3 / 2}$ is constant (as shown in our Appendix A), so that Eq. (7) can be evaluated at any radius $R_{0}$, provided it remains within the range where the adopted assumptions for the scaling of the stellar disk surface density remain valid $\left(R_{0} \lesssim 1 \mathrm{kpc}\right)$. The constant $\alpha\left(\eta_{\mathrm{k}}, \eta_{\mathrm{d}}\right) \sim 0.1-5$ is given in Eq. (A.14) and parameterizes the strong dependence of the normalization on the exact value of $\eta_{\mathrm{k}}$ of the Schmidt-Kennicutt law (see Appendix A). To maximize the contribution of the DI mode to the evolution of AGN, in the following we shall keep such a parameter to a maximum value $\alpha\left(\eta_{\mathrm{k}}, \eta_{\mathrm{d}}\right)=10$. Finally, following HQ11, we assume $R_{\text {acc }}$ to be equal to $\sim 10^{-2} R_{\mathrm{BH}}$.

Since our semi-analytical model uses exponential-law density profiles to describe the disk surface density profile (at galactic scales), we have also developed all the calculations for the case of "exponential disks". The resulting mass inflow rate is

$$
\begin{aligned}
\frac{\mathrm{d} M_{\mathrm{BH}}}{\mathrm{d} t} \approx & \alpha\left(\eta_{\mathrm{k}}\right) f_{\mathrm{d}}^{19 / 12}\left(\frac{M_{\mathrm{BH}}}{10^{8} M_{\odot}}\right)^{5 / 12}\left(\frac{M_{\mathrm{d}}\left(R_{0}\right)}{10^{9} M_{\odot}}\right)^{3 / 4} \\
& \times\left(\frac{R_{0}}{100 \mathrm{pc}}\right)^{-3 / 2}\left(\frac{R_{\mathrm{acc}}}{10^{-2} R_{\mathrm{BH}}}\right)^{5 / 6} M_{\odot} \mathrm{yr}^{-1}
\end{aligned}
$$

with $R_{\mathrm{d}}$ the exponential length of the disk, and all the calculations for exponential disks are shown in Appendix B. All the factors in Eq. (9) are the same of Eq. (7); $\alpha\left(\eta_{\mathrm{k}}, \eta_{\mathrm{d}}\right)=0.1-1.2$ (depending on $\eta_{\mathrm{k}}$ ) is now given by Eq. (A.14) with $\eta_{\mathrm{d}}=0$. With the choice $R_{\text {acc }} \sim 10^{-2} R_{\mathrm{BH}}$, the inflow is quite insensitive to the exact values of $R_{\mathrm{acc}}$ and $R_{\mathrm{BH}}$; however, when it is necessary (see next section), we eventually take $R_{\mathrm{acc}} \sim 0.1 \mathrm{pc}$ and $R_{\mathrm{BH}} \sim 10 \mathrm{pc}$.

\subsubsection{Computing the nuclear star formation rate}

Since the above models assume equilibrium between mass inflow and star formation, a star formation activity is always connected to the BH acccretion rate given in Eqs. (7) or (9), and can be computed in detail after Eq. (6), as shown in Appendix A. As a result, a star formation rate $\dot{M}_{*, \mathrm{DI}}=A_{*, \mathrm{DI}} \dot{M}_{\mathrm{BH}}$ is obtained, where the exact value of the proportionality constant $A_{*, \mathrm{DI}} \gtrsim 10^{2}$ depends on the detailed radial profile of the disk potential and of the gas surface density. The effects of changing $A_{*, \mathrm{DI}}$ within the limits derived in Appendices $\mathrm{A}$ and $\mathrm{B}$ will be quantitatively shown in the next section. Here we note that, given a total amount of baryons available for accretion, increasing $A_{*, \mathrm{DI}}$ leads to a faster gas consumption, and hence to a lower accretion rates at subsequent times, with the effect of decreasing the number of active AGNs. Thus, to investigate the maximum impact of the DI mode on the growth of BHs and on the evolution of AGNs it is sufficient to adopt proper lower limits for the DI star formation rate. This can be done within the framework of the DI model introduced above, and the derivation is shown (Appendix A, B) for both a power law and an exponential profile of the disk surface density. We obtain a lower limit $A_{*, \mathrm{DI}}=100$ (Eq. (A.18)) for a power-law profile (see Sect. A.5), corresponding to the contribution to the star formation from the inner region $R \lesssim R_{\mathrm{BH}}$. This also constitutes a lower limit for the case of an exponential disk, since in the inner region, the potential determining the mass inflow is dominated by the $\mathrm{BH}$ and does not depend on the assumed disk profile (see also Appendix B, Eqs. (B.11)-(B.13)). The contribution to the star formation rate from outer regions depends strongly on the assumed disk surface density; in the case of a power-law profile, larger and larger contributions are obtained when the power-law behaviour is extrapolated to regions far from the nucleus (see Eqs. (A.19)-(A.22)), while in the case of an exponential profile (more realistic in regions far from the $\mathrm{BH}$ sphere of influence $R \gg R_{\mathrm{BH}}$ ) we obtain an additional contribution saturating to $A_{*, \mathrm{DI}}=60$.

\section{Results}

We compute separately the effects of the two accretion modes on the AGN population. To compare with observations, the AGN bolometric luminosity is computed as

$L_{\mathrm{AGN}}=\eta c^{2} \frac{\mathrm{d} M_{\mathrm{BH}}}{\mathrm{d} t}$

where $\mathrm{d} M_{\mathrm{BH}} / \mathrm{d} t$ is taken from Eq. (3) for the IT mode, and from Eq. (7) (or Eq. (9)) for the DI models. We adopt an energyconversion efficiency $\eta=0.1$ (see Yu \& Tremaine 2002). The luminosities in the UV and in the X-ray bands are computed from the above expression using the bolometric correction given in Marconi et al. (2004).

We stress again that in the present work we do not attempt to find a best-fitting combination of the two accretion modes. Rather, we are interested in isolating the effects of the two accretion modes on the evolution of the AGN population, to single out the physical regimes where they may be effective in driving the AGN evolution. Given that in our previous paper (and in the following results), the IT mode has been shown to be able to provide acceptable fits to the observed evolution of the AGN population, our strategy will be to maximize the possible DI accretion rates in order to assess whether and for what kind of object we expect it to be competitive with the IT mode. To this aim, we explore the possible variants of the DI model provided by different choices for i) the normalization $\alpha\left(\eta_{\mathrm{K}}, \eta_{\mathrm{d}}\right)$ in Eq. (7), which in turn is related to the present uncertainties concerning the slope of the Kennicut-Schimdt law $\eta_{\mathrm{k}}$; ii) the different disk surface density profiles, determining the slope $\eta_{\mathrm{d}}$ (see Sect. 3.2.1) and the dependence of $\dot{M}_{\mathrm{BH}}$ on both the disk mass fraction and the gas fraction (through the boundary condition $A\left(R_{\mathrm{BH}}\right)$ in Appendix A; iii) the star formation associated to the DI mode and discussed in Sect. 3.2.2.

These quantities are not freely tunable, since they can only vary within limits set by observations and simulations. The former set a limit for the Kennicut-Schmidt index $3 / 2 \leq \eta_{\mathrm{k}} \leq 7 / 4$ 
(see Sect. 3.2.1). The latter provide a range of possible gas surface density profiles ranging from $\Sigma_{\mathrm{g}} \sim R^{-3}$ to $\Sigma_{\mathrm{g}} \sim R^{-1}$, bracketing the effects of the present uncertainties in the physics of the inter-stellar medium (see Hopkins \& Quataert 2010). These mainly concern the effective equation of state of the gas, which widely varies from cases where it is quite stiff on small scales (near-adiabatic, similar to what is adopted in the studies of Mayer et al. 2007; Dotti et al. 2009), through cases where the gas is allowed to cool to a cold isothermal floor and forms a clumpy, inhomogeneous medium (on galactic scales, similar to what is assumed in Bournaud et al. 2007; Teyssier et al. 2010), and includes cases where the gas motions gain a significant contribution from resolved turbulent motions, and the gas mass distribution can be highly inhomogeneous and clumpy. The above range of uncertainty for the gas surface density profiles translates (Eqs. (A.1), (A.2), (B.8)) into values ranging from $\eta_{\mathrm{d}}=1 / 2$ (the HQ11 case) to $\eta_{\mathrm{d}}=0$ (our exponential case).

For the above ranges of uncertainty $3 / 2 \leq \eta_{\mathrm{k}} \leq 7 / 4$ and $0 \leq \eta_{\mathrm{d}} \leq 1 / 2$, the normalization of the $\mathrm{BH}$ accretion rates in Eqs. (8) and (9) remains in the range $0.1 \lesssim \alpha\left(\eta_{\mathrm{k}}, \eta_{\mathrm{d}}\right) \leq 5$ (see Sect. 3.2.1). To investigate the maximum impact of DI, we adopt a safe normalization $\alpha\left(\eta_{\mathrm{k}}, \eta_{\mathrm{d}}\right)=10$, the same as is considered by HQ11 to constitute a definite upper limit. A final source of uncertainty comes from the contribution to the nuclear star formation rate from regions outside the $\mathrm{BH}$ sphere of influence $R \geq R_{\mathrm{BH}}$. Assuming it to be zero sets a lower limit $A_{*, \mathrm{DI}}=100$ for the normalization of the nuclear star formation rate, which maximizes the amount of inflowing gas feeding the $\mathrm{BH}$ accretion, as discussed in Sect. 3.2.2. We refer to the combination $\alpha=10$ and $A_{*, \mathrm{DI}}=100$ as "maximal" DI model. In addition, we also explore the effect of the additional contribution to the nuclear starbursts from regions far from the $\mathrm{BH}$ sphere of influence.

In sum, following the lines above, we explore three variants of the DI mode:

- a fiducial case corresponding to the model adopted by HQ11 (Eq. (7)), computed assuming a power-law disk surface density profile $\eta_{\mathrm{d}}=1 / 2$ with a maximized value of the parameter $\alpha\left(\eta_{\mathrm{K}}, \eta_{\mathrm{d}}\right)=10$ (see Sect. 3.2.1), and assuming the lower limit for the associated star formation rate $A_{*, \mathrm{DI}}=100$ (see Sect. 3.2.2 and Appendix A).

- the case of an exponential profile for the disk surface density (Eq. (9)), keeping the same values for $\alpha\left(\eta_{\mathrm{K}}, \eta_{\mathrm{d}}\right)=10$ and $A_{*, \mathrm{DI}}=100$ (see Sect. 3.2.2 and Appendix B).

- a model with enhanced star formation rate associated to DI (for a power-law profile). It assumes a normalization of the star formation associated to DI above the lower limits derived in Sect. 3.2.2, with an additional contribution $A_{*, \mathrm{DI}}=100$ from regions far from the $\mathrm{BH}$ sphere of influence $\left(R \gg R_{\mathrm{BH}}\right)$.

Having defined our reference set of DI cases, we proceed to study the evolution of the AGN population under different triggering models.

\subsection{Evolution of the AGN luminosity function and BH mass function}

The evolution of the AGN UV luminosity funtions predicted in the IT scenario is compared with the one corresponding to the DI mode in Fig. 4. The luminosity functions are computed in the UV band at $\lambda=1450 \AA$, where most of the UV rest frame data on AGN are collected and where the average AGN emission shows the peak in the spectral energy distribution, which is representative of the overall bolometric emission. The predictions are compared with data taken from different bands, including the X-ray data (corrected for obscuration) by La Franca et al. (2005); Ebrero et al. (2009) at low redshifts $z \lesssim 1.5$, the X-ray data for objects in the COSMOS field by Brusa et al. (2010); Civano et al. (2011); and the Chandra X-ray data for objects detected at $z \geq 4$ in the GOODS south field by Fiore et al. (2012). These are converted to the UV with the bolometric corrections in Marconi et al. (2004). Details on the conversion procedure are given in Fiore et al. (2012) and Giallongo et al. (2012).

The results shown in Fig. 4 indicate that none of the variants of the DI mode can provide the accretion needed to obtain high-luminosity QSO with $M_{1450} \leq-26$, especially at redshifts $z \gtrsim 3.5$. This is because the accretion rates in Eqs. (7) and (9) have a mild dependence on the BH mass, but depend very strongly on the disk mass fraction and on the gas mass fractions $\left(f_{\mathrm{d}}\right.$ and $\left.f_{\text {gas }}\right)$. Thus, in galaxies with a relatively low gas fraction, or in galaxies with a large $\mathrm{B} / \mathrm{T}$ ratio, the DI mode is more suppressed than the IT mode. As expected DI can instead be very effective in the range of galaxy masses (and AGN luminosities) dominated by disk galaxies $-25 \lessgtr M_{1450} \lesssim-22$. While we must keep in mind that the predicted DI luminosity functions in Fig. 4 constitute upper limits, the results show that in such a magnitude range the DI accretion rates could compete with - or even overtake - those attained in the IT scenario, at least for redshifts $z \lesssim 3.5$. At higher redshifts, although galaxies are surely gas rich with large $f_{\text {gas }} \sim 1$, the typical disk mass fraction $f_{\mathrm{d}}$ decreases since - in the standard scenario assumed in semianalytic models - the frequent major interactions continuously disrupt the forming disks.

Within the assumed modelling for the inflow driven by DI, our fiducial DI case constitutes an effective upper limit for the bright end of the luminosity function. Increasing the associated star formation $A_{*, \mathrm{DI}}$ above the lower limit $A_{* \text { DI }}=100$ would yield lower luminosity functions, as shown in Fig. 4, for the reasons explained in Sect. 3.2.2. Changing the disk power-law density profile assumed in the original version of HQ11 to an exponential profile also results in a lower abundance of bright AGN. Indeed, the same fiducial model must be regarded as an effective upper limit, since the assumed value for the normalization $\alpha\left(\eta_{\mathrm{K}}, \eta_{\mathrm{d}}\right)=10$ is actually appreciably higher than any value attained with realistic values of $\eta_{\mathrm{k}}$ and $\eta_{\mathrm{d}}$, as shown in Appendix A (see Eq. (A.14) and the text below).

For the IT model, the results presented here are very similar to those obtained in the earlier version of our model (see, e.g., Giallongo et al. 2012), and show an acceptable matching with the data. For $z \geq 4.5$ the real size of the model over-prediction with respect to the faintest data points still has to be assessed. In fact, the observations by Fiore et al. (2012) could be affected by several uncertainties and significant incompleteness. The objects have been selected from very deep Hubble Space Telescope near infrared images at $H \approx 27$ and measured at the faintest X-ray fluxes of $F_{X} \approx 2 \times 10^{17} \mathrm{erg} \mathrm{s}^{-1} \mathrm{~cm}^{-2}$. At the lowest $\mathrm{X}$-ray luminosities, objects with relatively high X/optical ratio, and consequently with $H>27$, are lacking in their survey. As a consequence, uncertainties in the Fiore et al. (2012) luminosity functions data due to systematic errors could be larger than shown in the figure based on number statistics.

While the above result strongly disfavour DIs as the dominant accretion mode in high-luminosity AGNs, they also indicate that it could be as effective as the IT mode (or even more) in intermediate-luminosity AGNs especially at intermediate redshifts $2 \lesssim z \lesssim 3.5$. This is the epoch where massive star formation - possibly triggered by DI in gas-rich objects - is observed in disk galaxies (Bournaud et al. 2007; Genzel et al. 2008). Thus, we can seek proper observables able to assess whether one of 
N. Menci et al.: Triggering active galactic nuclei in hierarchical galaxy formation: disk instability vs. interactions

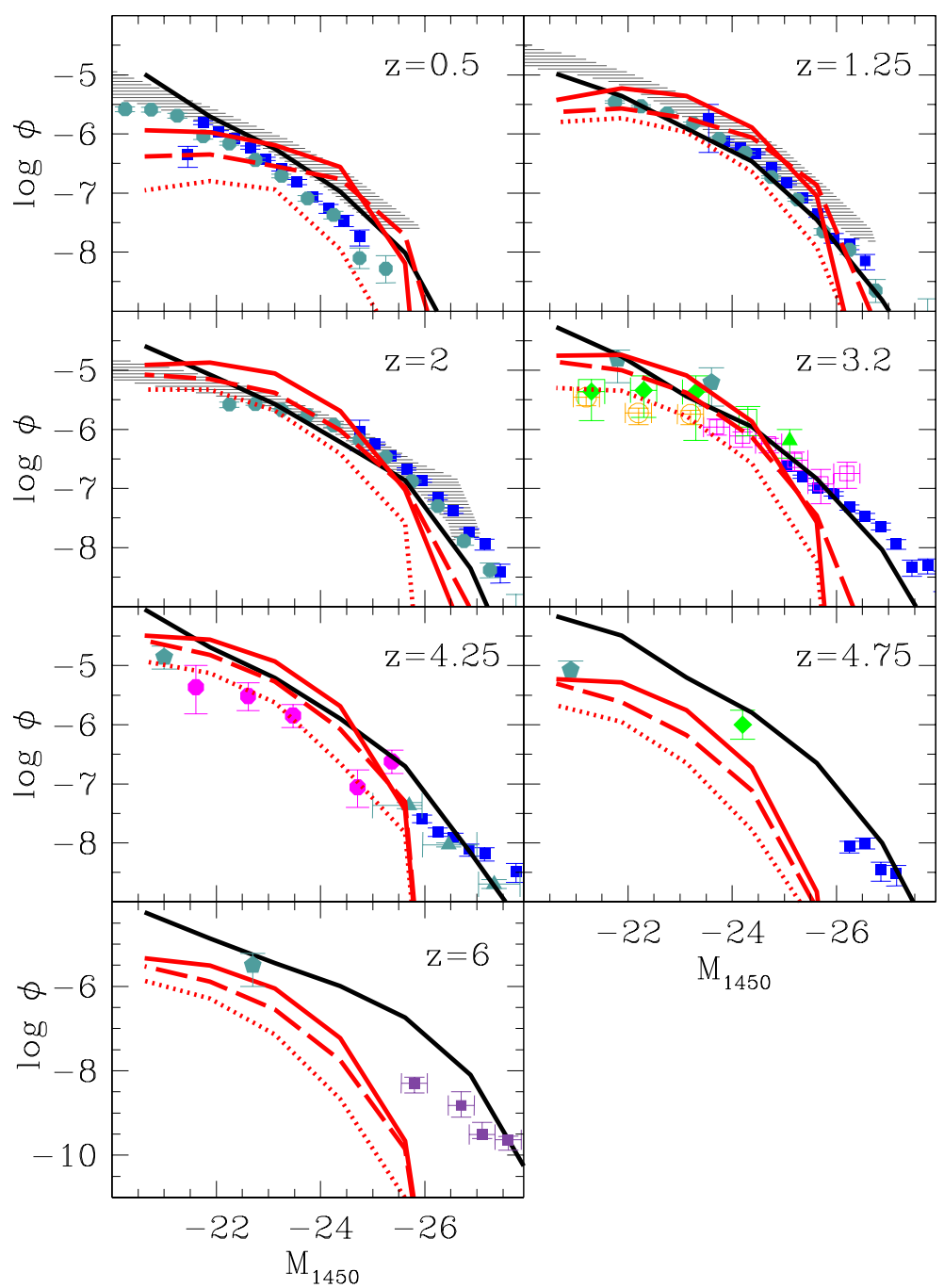

Fig. 4. Predicted evolution of the AGN luminosity function in the UV band (at $1450 \AA$ ). Solid black lines refer to the IT scenario, and red solid lines to the DI model. We also plot variants of the DI model: dashed lines refer to the DI model computed with an exponential profile, while dotted lines to the DI model with increased star formation (see text). We compare with observed UV luminosity functions by Richards et al. (2006, solid squares), Croom et al. (2009, solid circles at $z<3$ ), Siana et al. (2008, open squares), Bongiorno et al. (2007, open circles), Fontanot et al. (2007, solid diamonds), Glikman et al. (2011, big filled circles at $z=4.25$ ), Jiang et al. (2009; solid squares at $z \geq 6$ ). We also include luminosity functions from $\mathrm{X}$-ray observations, converted to UV through the bolometric corrections in Marconi et al. (2004): the shaded region brackets the determinations derived in the X-ray band at $z \leq 2$ by La Franca et al. (2005), Ebrero et al. (2009), Aird et al. (2010), while at higher redshifts we plot data from Fiore et al. (2012, penthagons), Brusa et al. (2010, triangle), Civano et al. (2011, green empty squares). the two modes clearly dominates in this luminosity and redshift range.

A possible approach could be to seek for signatures in the $\mathrm{BH}$ mass function and/or in the $M_{\mathrm{BH}}-M_{*}$ relation. The former statistically describes the growth of $\mathrm{BH}$ masses corresponding to the evolution of the $\mathrm{BH}$ accretion rates shown in Fig. 4. Our predictions for such a quantity are presented for both the IT and the fiducial DI models in Fig. 5. At low redshifts the predictions are compared with a compilation of data (Shankar et al. 2009). While current data do not allow to distinguishing between the DI and the IT scenario at low redshift $z \lessgtr 0.3$, the predicted evolution of the $\mathrm{BH}$ mass funtions differs appreciably at higher redshifts; in particular, as expected from the evolution of the AGN luminosity functions, DI provide a much lower abundance of massive $\mathrm{BH}$ at $z \gtrsim 1$.

\subsection{The $M_{B H}-M_{*}$ relation}

A larger difference between the IT and the DI prediction is shown by the local $M_{\mathrm{BH}}-M_{*}$ relation, shown in Fig. 6 . While both the IT and the DI accretion modes provide a local relation consistent with observations, the slope and - most of all - the scatter differ appreciably. While present data do not allow a clear preference for one of the two modes, it is interesting to note that the large scatter characteristic of the IT mode comes from the variance in the merging histories, which has a strong impact in a model where interactions provide the trigger and the power of the AGN emission; the origin of such a variance can be visualized through the paths followed by AGN host to reach their final position in the $M_{\mathrm{BH}}-M_{*}$ plane.

Such paths include situations in which the progenitor of present-day massive $\mathrm{BH}$ are characterized by an early accelerated phase of $\mathrm{BH}$ growth, which brings them above the local relation at early times $z \gtrsim 4$, followed by a later phase where the BH growth appreciably decelerates while the "quiescent" star formation gradually increases the stellar content of their host galaxies, and these paths are those leading to the early building up of large-mass BHs at high redshifts $z \gtrsim 4$. The paths passing below the local relation instead correspond to galaxies retaining a large fraction of their gas down to late cosmic epoch, so that they form stars at very high rates down to $z \approx 2$ (the most star forming objects are those corresponding to SMG, see Lamastra et al. 2010). Such a large variance in the paths leading to the local relation is missing in the DI scenario, where the trigger and the accretion rate of AGN are provided by internal properties which are less prone to the merging history. Indeed, in the DI the paths are confined in a small region of the plane; the growth of BH follows a corresponding growth of the stellar mass content, and phases where either $\dot{M}_{\mathrm{BH}}$ or $\dot{M}_{*}$ largely dominate are absent. This means that we expect a tight correlation between the AGN luminosity and the star formation rate in the host galaxy. This 
A\&A 569, A37 (2014)

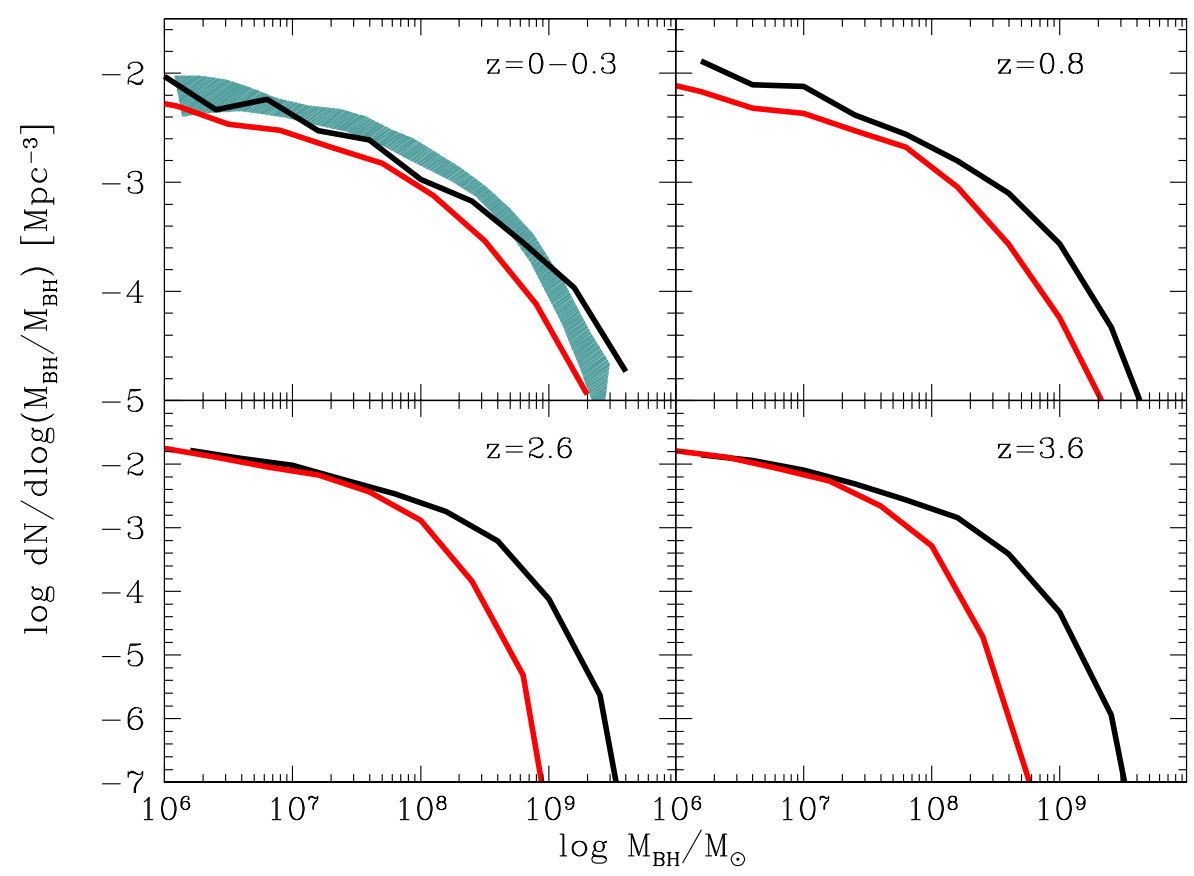

Fig. 5. Predicted evolution of the BH mass function in the IT scenario (solid black lines) and in the DI model (solid red line). The shaded region defines the spread in observational estimates obtained using different methods, as compiled by Shankar et al. (2009). constitutes an interesting point that we shall investigate in detail in a following paper.

\subsection{The distributions of the Eddington ratio}

The different paths characterizing the $\mathrm{BH}$ growth in the IT and DI models suggest that the specific growth rate $\dot{M}_{\mathrm{BH}} / M_{\mathrm{BH}}$ could constitute an effective quantity for distinguishing the two modes; thus, we investigated how the distributions of Eddington ratio $\lambda=L_{\mathrm{AGN}} / L_{\mathrm{Edd}}$ (here $L_{\mathrm{Edd}} \propto M_{\mathrm{BH}}$ is the Eddington luminosity, and $L_{\mathrm{AGN}} \propto \dot{M}_{\mathrm{BH}}$ after Eq. (10)) compares with present observations. The result is presented in Fig. 7, where the AGN bolometric luminosity is shown as a function of the $\mathrm{BH}$ masses for the IT and DI scenarios, in two redshift bins. Luminosities corresponding to Eddington ratios $\lambda=1, \lambda=10^{-1}$, and $\lambda=10^{-2}$ are also shown. Here the distributions appear substantially different when the IT or the DI modes are assumed. In the former case, a wide spread is present for most of the explored luminosity range, while the average value of $\lambda$ increases with increasing $L_{\mathrm{AGN}}$, to reach values $\lambda \approx 1$ at the highest luminosities. Conversely, for the DI scenarios the distributions are characterized by average values of $\lambda$ increasing for decreasing luminosities and by a small scatter. This is expected, owing to the tighter region of the parameter space (gas rich $f_{\text {gas }} \sim 1$, disk dominated $f_{\mathrm{d}} \sim 1$ dominated galaxies) where the DI mode is expected to be effective after Eq. (7), and also to the smaller variance associated to the merging histories when an internal trigger is assumed, as we discussed above. We compare our predictions with data from Rosario et al. (2013a,b), derived from spectroscopically observed AGN in the COSMOS field at $0.5<z<2.2$ with reliably BH mass estimates from multiple optical spectroscopic surveys: the Sloan Digital Sky Survey (DR7; Abazajian et al. 2009), the zCOSMOS bright and deep surveys (Lilly et al. 2007), Magellan/IMACS program (Trump et al. 2009). BH masses were computed using virial relationships and $\mathrm{H} \beta$ and MgII emission lines (Trakhtenbrot \& Netzer 2012), while bolometric luminosities were estimated using bolometric corrections to the monochromatic luminosities at either $5100 \AA$ or $3000 \AA$ rest frame. The choices of bolometric corrections are derived in Trakhtenbrot \& Netzer (2012) and are consistent with the prescriptions of Marconi et al. (2004).

We note that at low AGN luminosities the comparison with data is affected by the observational bias set by the flux limit of the sample. This corresponds to $L_{\mathrm{AGN}} \sim 10^{44.2} \mathrm{erg} / \mathrm{s}$ at $z=0.5-1$ and $L_{\mathrm{AGN}} \sim 10^{44.7} \mathrm{erg} / \mathrm{s}$ at $z=1.5-2$. At low redshift $z=0.5-1$, where the flux limit could still allow the low-luminosity end of the distribution to be probed, the comparison with the model is affected by the paucity of data points. This is due to a further selection bias, since the zCOSMOS AGN, which statistically dominate the sample, were specifically chosen to include the MgII line, which enters into the wavelength range of the zCOSMOS bright spectra at $z \sim 1$.

Since the distribution of AGN in the $L_{\mathrm{AGN}}-M_{\mathrm{BH}}$ plane is appreciably different for the DI and IT cases, a possible way of determining which of the two modes dominates the accretion for intermediate- and low-luminosity AGN could consist in comparing the corresponding distributions of Eddington ratios $\lambda$ with present observations at low redshifts, where low-luminosity objects are accessible to observations; in fact, for such AGN, the IT mode is expected to yield distributions with larger variance and skewed to values $10^{-2} \lesssim \lambda \lesssim 10^{-1}$, while higher values of $\lambda$ and less scatter are expected for the DI mode.

However, when the model predictions for the two modes are compared with the data (see Fig. 8) we face with a complex situation; in fact, the observational distributions (at least) depend on i) the method adopted to select AGN; ii) the adopted bolometric correction; iii) the method adopted to measure the BH mass (for a discussion of the above points, see, e.g., Netzer et al. 2009a,b and references therein). This is illustrated in the top left-hand panel, where we compare with low-redshift data for AGN with $L_{\mathrm{AGN}}<10^{4} 6 \mathrm{erg} \mathrm{s}^{-1}$ taken from Hickox et al. (2009) and Kollmeier et al. (2006). The former refer to AGN at $0.25<z<0.8$ selected in different wavebands. The radio data are taken from the Westerbork Synthesis Radio Telescope $1.4 \mathrm{GHz}$ radio survey (de Vries et al. 2002), the X-ray data are from the XBootes survey, covering the full AGES (AGN and Galaxy Evolution Survey) spectroscopic region (Murray et al. 2005; Kenter et al. 2005), while the mid-infared data are from 
N. Menci et al.: Triggering active galactic nuclei in hierarchical galaxy formation: disk instability vs. interactions
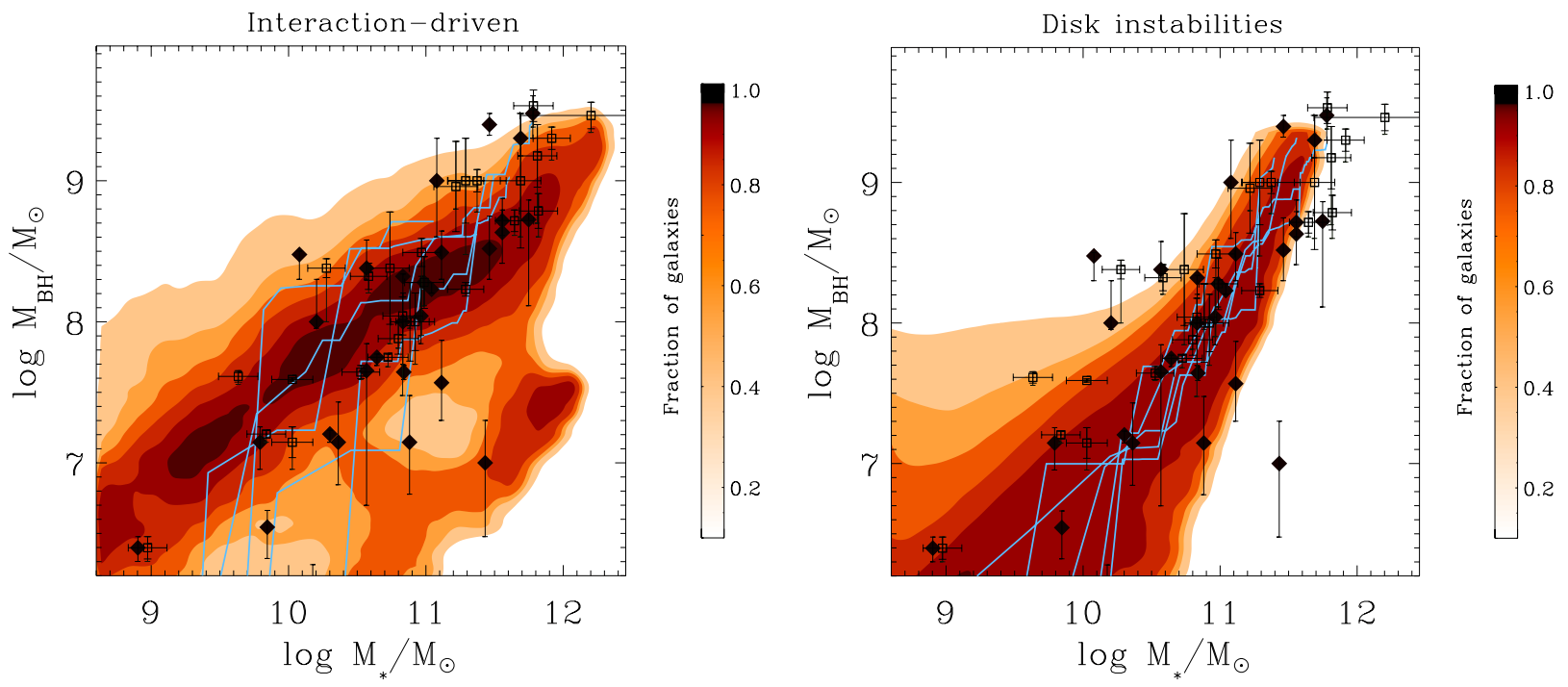

Fig. 6. Local $M_{\mathrm{BH}}-M_{*}$ relation for the IT model (left panels) and the DI model (right panels). Data points represent the observed local relation from Häring \& Rix (2004, diamonds), and Marconi \& Hunt (2003, squares, here $M_{*}$ is derived using the best-fitting virial relation of Cappellari et al. 2006); the colour code represents the fraction of AGN as a function of $M_{\mathrm{BH}}$ for any given value of $M_{*}$, as indicated by the colour bar. We also show some of the paths in the $M_{\mathrm{BH}}(t)-M_{*}(t)$ plane followed, during their evolution, by BHs (and by their host galaxies) reaching a final mass of $M_{\mathrm{BH}}(z=0) \geq 10^{9} M_{\odot}$.
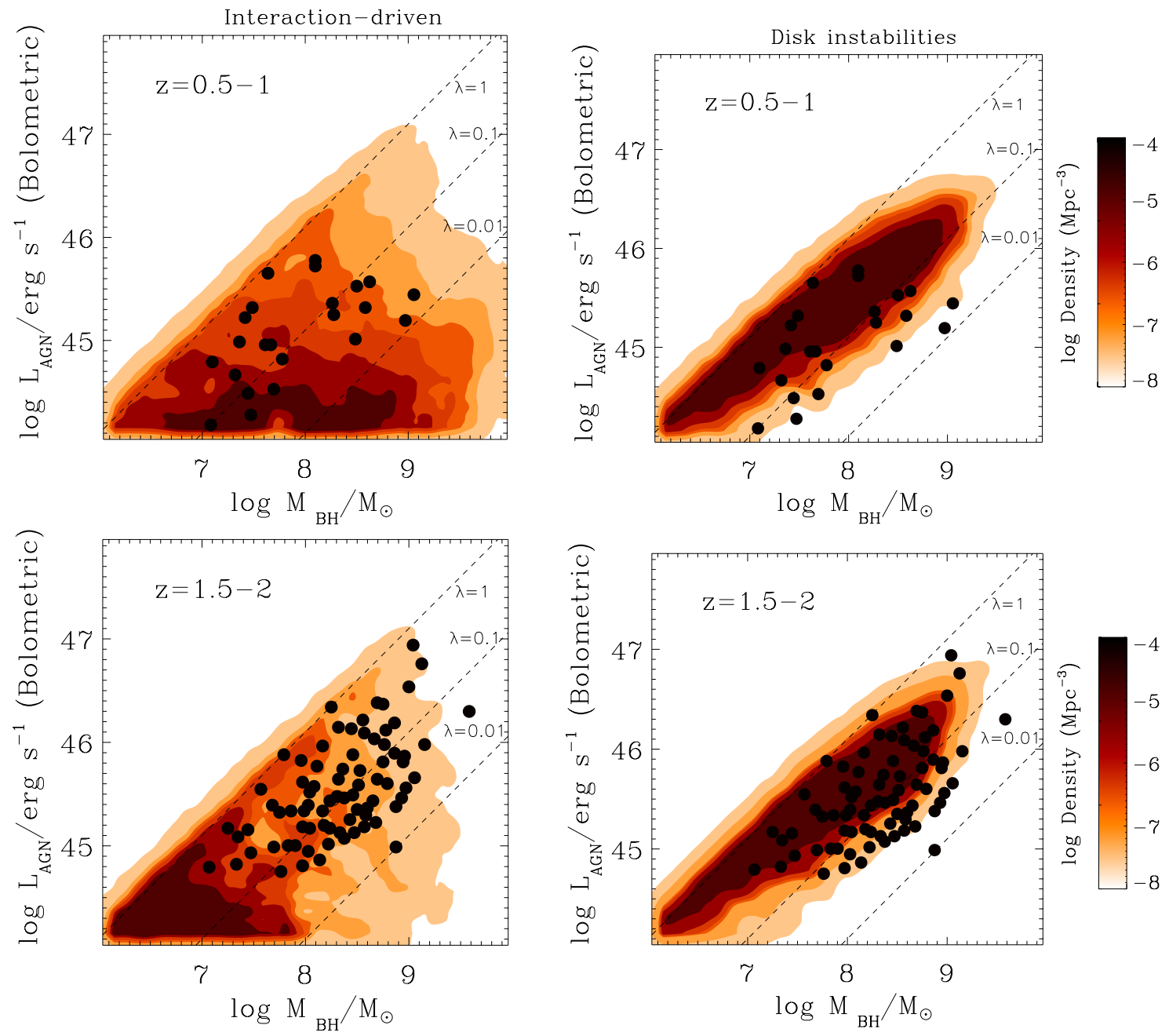

Fig. 7. Predicted distribution of AGN in the $L_{\mathrm{AGN}}-M_{\mathrm{BH}}$ plane for the IT (left) and DI (right) models is compared with data from Rosario et al. $(2013 \mathrm{a}, \mathrm{b})$ in two redshift bins. The lines corresponding to Eddington ratio $\lambda=1, \lambda=0.1$ and $\lambda=0.01$ are also indicated. 

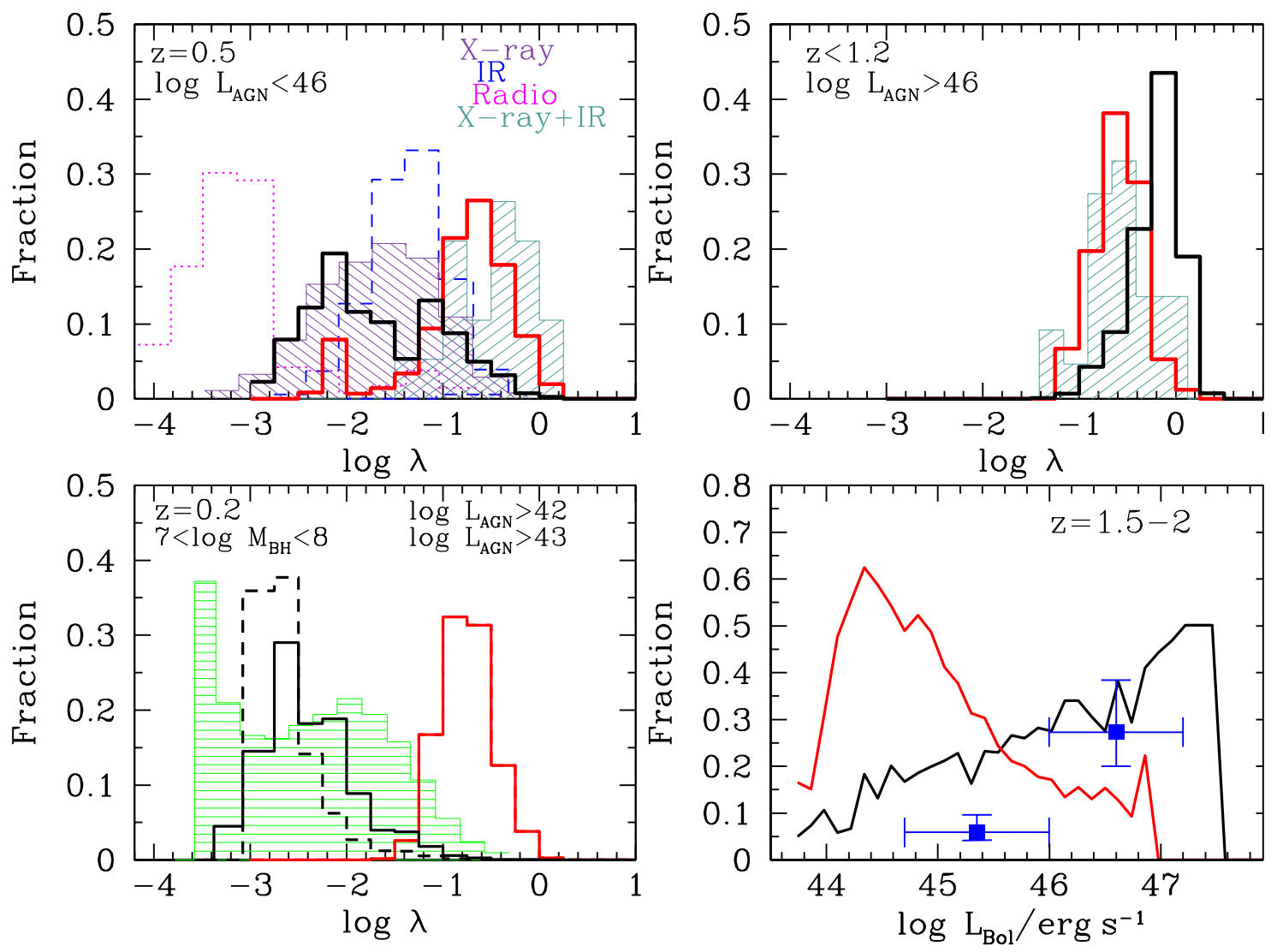

Fig. 8. Top left panel: predicted distributions of the Eddington ratio $\lambda$ in the IT (solid black line) and DI (solid red line) scenarios for AGN with bolometric luminosity $L_{\mathrm{AGN}}<10^{46} \mathrm{erg} \mathrm{s}^{-1}$ at $z \leq 0.5$. They are compared with different sets of data (see text for details): a sample of X-ray-selected AGN (dashed violet histogram), of IR-selected (dashed histogram) and Radio selected AGN (dotted histogram) from Hickox et al. (2009), and the sub-sample of X-ray and IR AGN from Kollmeier et al. (2006, dark green histogram) corresponding to $z \leq 0.5$. Top right panel: as above, but for luminous AGN with $L_{\mathrm{AGN}}>10^{46} \mathrm{erg} \mathrm{s}^{-1}$ at $z \leq 1.2$. The dashed histogram represents observational results from Kollmeier et al. (2006). Bottom left panel: as above, but for AGN with BH masses in the range $10^{7} M_{\odot} \leq M_{\mathrm{BH}} \leq 0^{8} M_{\odot}$. Here we compare with data from Kauffmann \& Heckman (2009, histogram). Bottom right panel: fraction of AGN with high Eddington ratios $\lambda \geq 0.5$ as a function of the AGN bolometric luminosity $L_{\mathrm{AGN}}$, at $z=2$. As above, the solid black line corresponds to the IT mode and the solid red line to the DI case. The points are computed from the data by Rosario et al. (2013a,b) shown in Fig. 7.

the Spitzer IRAC Shallow Survey covering the full AGES field in all four IRAC bands (Eisenhardt et al. 2004). The authors base their estimate $M_{\mathrm{BH}}$ on the $L_{B \text {,bul }}-M_{\mathrm{BH}}$ relation after estimating the bulge luminosity of the host galaxy in the $B$ band, while the bolometric luminosity for X-ray AGN are derived from the bolometric correction of Hopkins et al. (2007); for radio AGNs that are not detected in X-rays, they used the X-ray stacking results to derive approximate upper limits on the X-ray AGN luminosity, while for IR AGNs that are not detected in X-rays, $L_{\mathrm{bol}}$ is derived from the rest-frame $4.5 \mu \mathrm{m}$ luminosity. We also compare this with data from Kollmeier et al. (2006), derived from the AGES survey and consisting of X-ray (XBootes survey, Murray et al. 2005, Kenter et al. 2005) or mid-infrared (Eisenhardt et al. 2004) point sources with optical magnitude $R \leq 21.5 \mathrm{mag}$ and optical emission-line spectra characteristic of AGNs. Black hole masses were estimated using virial relationships and $\mathrm{H} \beta \mathrm{MgII}$, and CIV emission-line widths, while bolometric luminosities were estimated using the bolometric correction of Kaspi et al. (2000) to the monochromatic luminosities at $5100 \AA$.

The comparison in Fig. 8 (top-left panel) shows that, while a marked difference is indeed apparent between model predictions for the IT mode and the DI mode, the observational distributions for X-ray-selected AGN (Hickox et al. 2009) extend to lower values of $\lambda$ with respect to IR AGN, while radio-selected objects show a distribution peaked to much lower values of $\lambda$. While the X-ray homogeneous sample in Hickox et al. (2009) would favour the IT accretion mode, the mixed IR $+\mathrm{X}$-ray data by Kollmeier et al. (2006) are instead best matched my the DI mode predictions, showing the impact of adopting different methods to measure $\mathrm{BH}$ masses on the comparison with model predictions. Again scatter constitutes a crucial diagnostics for pinning down the dominant accretion mode for low- and intermediateluminosity AGN (see also Shankar et al. 2013). Much weaker constraints come instead from luminous AGN (top-left panel), where the two modes yield similar distributions and scatter. For luminous AGN, however, we know from the results in Fig. 4 that IT mode must dominate, since accretion from DI does not provide the observed abundance of bright AGN.

We also compare (Fig. 8, bottom left panel) with data for low-mass BHs $\left(10^{7} \leq M_{\mathrm{BH}} / M_{\odot} \leq 10^{8}\right)$ taken from Kauffmann \& Heckman (2009). Their sample is drawn from the Data Release 4 of the SDSS at $z \simeq 0.2$ (York et al. 2000; AdelmanMcCarthy et al. 2006). AGN are identified from their position in the $[\mathrm{O}$ III $](\lambda=5007 \AA) / \mathrm{H} \beta$ versus $[\mathrm{N} \mathrm{II]}(\lambda=6583 \AA) / \mathrm{H} \alpha$ diagram, as discussed in Kauffmann et al. (2003). Here $L_{\mathrm{AGN}}$ is derived from the luminosity $L_{\text {OIII }}$ of the $[\mathrm{OIII}](\lambda=5007 \AA)$ line after applying a bolometric correction that they estimate in the range 500-800, while $\mathrm{BH}$ masses were estimated using the 
stellar velocity dispersions measured from the SDSS spectra and the formula given in Tremaine et al. (2002). Within the limitations of available data - affected by substantial uncertainties concerning the bolometric correction, the measurements of the BH mass, and selection effects - the spread and the peak of the observed distribution seem to favour the IT scenario, but conclusive evidence is still missing. Also $\lambda$-distributions with a large spread are indicated by analysis of the observed AGN duty cycles (see Shankar et al. 2013).

Thus, at present the observational situation does not allow defining a definite distinction between the DI and the IT accretion modes. The key point is to assess whether low- and intermediate- luminosity AGN have a narrow distribution of $\lambda$ peaked at $\lambda \sim 1$ (as predicted for the DI mode) or a wider distribution extending to $\lambda \sim 10^{-1}-10^{-2}$. Thus, a straightforward observational test is constituted by the fraction of AGN with high $(\lambda \geq 0.5)$ Eddington ratios as a function of the AGN luminosity (choosing a redshift bin where significant statistics is ensured, $2 \leq z \leq 3$ ). The opposite behaviour is then predicted when IT and DI are assumed as dominant accretion modes, as shown in the bottom right-hand panel of Fig. 8. We stress again that, while the dominance of the IT mode for bright AGN results from the luminosity functions in Fig. 4, the interesting test is constituted by the low-luminosity region of the plot, where an increasing fraction for lower luminosities would clearly point towards the effectiveness of DI in triggering AGN; however, present data (taken by integrating the $L_{\mathrm{AGN}}-M_{\mathrm{BH}}$ data by Rosario et al. 2013a,b shown in Fig. 7) - although consistent with a dominance of the IT mode for luminous AGN - still do not allow probing the low-luminosity range. Measuring such a quantity and comparing it with the predicted distributions could provide a clear observational test to assess whether DI may be the dominant accretion mode for intermediate-low AGN luminosities.

\section{Discussion and conclusions}

Using a a state-of-the-art semi analytic model for galaxy formation, we have made a detailed investigation of the effects of accretion triggered by disk instabilities (DI) in isolated galaxies on the evolution of the AGN population and on the corresponding growth of massive BHs. Specifically, we took on, developed, and expanded the Hopkins \& Quataert (2011, HQ11) model for the mass inflow following disk perturbations, based on a physical description of nuclear inflows and tested against aimed $N$-body simulations.

We compared the evolution of AGNs due to this DI accretion mode with that arising in a scenario where galaxy interactions produce the sudden destabilization of large quantities of gas feeding the AGN (interaction-triggered - IT - mode). This constitutes the standard AGN feeding mode implemented in the earliest versions of our semi-analytic model, and has was extensively tested in our previous works. Here we did not attempt to develop a best-fitting model including both accretion modes, but rather we investigated the effects of assuming DI or IT as dominant modes studying the maximal contribution of DI to the evolution of the AGN population, within the framework of the HQ11 model for nuclear inflow. To this aim, we extended and developed the HQ11 model for DI to assess the effects of changing the assumed disk surface density profile and the associated nuclear star formation rates within the limits provided by $\mathrm{N}$-body simulations. The goal was to investigate how often and to what extent the galaxy properties evolving in a cosmological context (and described through a semi-analytic model) provide the nuclear inflows associated to disk instabilities in ultra-high resolution simulations. We obtained the following results:

- For luminosity $M_{1450} \gtrsim-26 \mathrm{AGN}$, the DI mode can provide the BH accretion needed to match the observed AGN luminosity functions up to $z \approx 4.5$. In such a luminosity range and redshift, it constitutes a viable candidate mechanism for fueling AGN, and can compete with the IT scenario as the main driver of the cosmological evolution of the AGN population.

- The DI scenario cannot provide the observed abundance of high-luminosity QSO with $M_{1450} \leq-26 \mathrm{AGN}$, as well as the abundance of high-redshift $z \gtrsim 4.5$ QSO with $M_{1450} \leq-24$. This is because the strong dependence of the $\mathrm{BH}$ accretion rate on the gas fraction $f_{\text {gas }}$ and on the $B / T$ ratio in the HQ11 model limits the effectiveness of such an accretion mode in massive galaxies; at high redshifts, the frequent major mergers predicted to take place at high redshifts $z \gtrsim 5$ in the host galaxies cause the rapid destruction of disks and then lower the accretion rate in the DI mode due to its strong dependence on the disk mass fraction $f_{\mathrm{d}}$. As found in our previous works, the IT scenario provides an acceptable match to the observed luminosity functions up to $z \approx 6$.

- The above conclusions concerning the DI mode do not change when a different (exponential) surface density profile is assumed for the disk with respect to the original HQ11 model, or when nuclear star formation rates higher than the lower limits derived in the framework of the HQ11 model are assumed. Indeed, the latter effect decreases the cold gas supply in the host galaxies and hence suppresses the effect of the DI accretion rates, leading to lower luminosity functions.

- The BH mass distribution predicted in the DI and IT scenarios at $z \lesssim 0.3$ are consistent with present observational constraints, although the IT model provides a better fit to the high-mass end. Also, the observed local $M_{\mathrm{BH}}-M_{*}$ relation is consistent with what is predicted in both the DI and IT scenarios. However, the DI scenario provides a slightly steeper slope of the relation, and, most of all, an appreciably smaller scatter. Such a difference comes from the larger variance in the paths followed by the AGN hosts to reach their final position in the $M_{\mathrm{BH}}-M_{*}$ plane in the IT scenario; in fact, in such a case, the trigger and the size of the $\mathrm{BH}$ accretion directly depend on the merging histories and are thus heavily affected by the associated variance. On the contrary, in the DI case the paths are confined to a small region of the plane. The growth of $\mathrm{BH}$ follows a corresponding growth of the stellar mass content, and phases where either $\dot{M}_{\mathrm{BH}}$ or $\dot{M}_{*}$ largely dominate are absent. This means that we expect a tight correlation between the AGN luminosity and the star formation rate in the host galaxy. This constitutes an interesting point that we shall investigate in detail in a subsequent paper.

- The distribution of the Eddington ratio $\lambda$ can constitute an effective probe to pin down the dominant fueling mechanism of AGN in the low-intermediate luminosity range $L_{\mathrm{AGN}}<$ $10^{46} \mathrm{erg} \mathrm{s}^{-1}$, where the DI and the IT modes are both viable candidates as the main drivers of the AGN evolution. In fact, for these AGN, the IT mode is expected to yield distributions with larger variance and skewed to values $10^{-2} \lesssim \lambda \lesssim 10^{-1}$, while higher values of $\lambda$ and a much smaller scatter are expected for the DI mode. We note that tuning the DI mode so as to yield lower values for the Eddington ratio $\lambda$ would result in a severe under-prediction of the abundance of AGN. The comparison of the model predictions with observational $\lambda$ distributions at low redshifts, where the population of low-luminosity is accessible, is still plagued by the combination of selection 
effects and uncertainties concerning the bolometric corrections and the adopted method pf measuring the BH mass, so current data still do not allow drawing firm conclusions. To alleviate the effects of the above biases and uncertainties by increasing the statistics, we propose a test at $z \gtrsim 2$ based on the fraction of low-luminosity AGN $\left(L_{\mathrm{AGN}} \leq 10^{44} \mathrm{erg} \mathrm{s}^{-1}\right)$ with high Eddington ratios $\lambda \geq 0.5$ at $z \gtrsim 2$. The presence of a peak at low luminosities $\left(L_{\mathrm{AGN}} \lesssim 10^{44} \mathrm{erg} \mathrm{s}^{-1}\right.$ ) would indicate a significant contribution of the DI mode to the growth of supermassive BHs. Finally, we note that our results for the small scatter of the $\lambda$-distribution in the DI scenario compared to the IT mode agree with those obtained from semi-empirical studies by Shankar et al. $(2012,2013)$.

We stress that the DI and IT modes actually constitute schematic representations of the actual AGN fueling process. The first case applies to isolated galaxies, where perturbations $a \approx 0.1-0.3$ of the potential $\Phi_{a}=a \Phi_{0}$ with respect to the axisymmetric thin disk potential $\Phi_{0}$ develop when the gas mass exceeds a critical value (Eq. (4)); in this case, the HQ11 model describes the corresponding mass inflow attainable in the galaxy nucleus. On the other hand, the IT model corresponds to a scenario where the large quantities of gas destabilized during encounters cannot be regarded as disk perturbations, and derives the corresponding nuclear feeding from the model by Cavaliere \& Vittorini (2000). Of course, intermediate situations may arise in minor merging events, where the gas destabilized during encounters or fly-by can be regarded as a perturbation of the potential. This makes it subject to the accretion limits give by the HQ11 model, which constitutes an interesting development of the model that we shall explore in a later paper.

To what extent do the HQ11 description implemented in our semi-analytic model represent the observed features generally associated to disk instabilities? As for the star formation properties of DI hosts, Silverman et al. (2009) have studied the correlation between $\dot{M}_{\mathrm{BH}}$ and the total star formation in galaxies hosting a AGN in isolated (non-merging) systems. Using a large sample of galaxies from the zCOSMOS survey, they selected a sample of moderately luminous AGN with no structural signs of recent interactions with other galaxies, so the best candidates to probe the DI scenario. They find that the median ratio $\dot{M}_{*} / \dot{M}_{\mathrm{BH}} \approx 10^{2}$ almost constant in the redshift range $z \sim 0.5-1$. This ratio is in good agreement with $A_{*, \mathrm{DI}}=100$ of our fiducial DI case computed in Appendix on the basis of the HQ11 model and with the results of the simulations by Hopkins \& Quataert (2010) and Bournaud et al. (2011).

As for the morphological properties of AGN in the DI scenario, Bournaud et al. (2012) compare a sample of 14 clumpy galaxies at $z=0.7$ with a sample of 13 stable and smoother galaxies at the same redshift. Since those clumpy galaxies are found to be on the main sequence of star formation and do not show clear signs of recent interactions, the authors infer that DI rather than mergers may be responsible for this activity. Using emission line diagnostic, they find that clumpy disks have a higher probability of containing moderately luminous AGN (bolometric luminosity between $10^{43} \mathrm{erg} \mathrm{s}^{-1}$ and $10^{44} \mathrm{erg} \mathrm{s}^{-1}$. Since the most powerful accretion episodes driven by DI are thought to be associated with clumpy morphologies and high gas fractions (see, e.g., Ceverino et al. 2010; Bournaud et al. 2011), the observed morphologies of low-intermediate AGN hosts are consistent with those expected in the DI description implemented here (Sect. 3.2.1). Thus, the description of DI adopted here and implemented in our semi-analytical model seems to capture the morphological and star formation signatures generally associated to AGN powered by DI.

How robust are our conclusions concerning the inefficiency of DIs in powering bright QSOs? The drop in the effectiveness of DI in accounting for bright AGNs is due to the strong dependence on the $\mathrm{B} / \mathrm{T}$ ratio (or, equivalently, on $f_{\mathrm{d}}$ ) in Eqs. (7) and (8). Such a dependence has been measured in simulations in a wide range of initial conditions and of galaxy properties (such as equation of state of the inter-stellar medium, and supernovae feedback, see Hopkins \& Quataert 2010), so it seems to constitute a robust feature of DI accretion mode. In addition, we adopted values $a=0.3$ for the amplitude of disk disturbances in the DI case. This actually constitutes an upper limit to values measured in simulations, which range from $10^{-2}$ to $3 \times 10^{-1}$ (Hopkins \& Quataert 2010), so again the effects of DI on the luminosity functions in Fig. 4 are actually upper limits. Indeed, Bournaud et al. (2011) study disk galaxies at $z \approx 2$ using both analytical and numerical estimates for the accretion rate onto the central BH in galaxies with high gas surface density continuously fed by gas streams. In their aimed and isolated galaxy simulations, they start from highly unstable $(Q<1)$ and gasrich disks $\left(f_{\text {gas }} \sim 0.5\right)$. The gas reservoir is supposed to be continuously fed by cold gas streams, and gravitational instability plays a crucial role in feeding the central $\mathrm{BH}$. Assuming $M_{\mathrm{BH}} / M_{\mathrm{blg}} \sim 3 \times 10^{-3}$ at $z \sim 2$, for a galaxy of barionic mass $10^{11} M_{\odot}$ and $M_{\mathrm{BH}} \sim 10^{8} M_{\odot}$, they obtain $\dot{M}_{\mathrm{BH}} \sim 0.04 M_{\odot} \mathrm{yr}^{-1}$ from their analytic estimate and a corresponding bolometric luminosity $L_{\mathrm{AGN}} \approx 2 \times 10^{44} \mathrm{erg} \mathrm{s}^{-1}$, corresponding to modest $\mathrm{X}$-ray luminosities $L_{X} \approx 10^{42}-10^{43} \mathrm{erg} \mathrm{s}^{-1}$. This is also the range of luminosities where the observational support of the relevance of DI in isolated galaxies is stronger, as noted above (see, e.g., Silverman et al. 2009; Bournaud et al. 2011). It is interesting to note that simulations suggest that the accretion episodes triggered by DIs is spread over a longer period (up to 10 times) compared to IT events (Bournaud et al. 2011); this constitutes an interesting issue that we plan to take on in a another paper.

We have also investigated the robustness of our results to changes in the modelling of the bulge growth during the nuclear starbursts associated to the gas inflows. Since all the inflowing gas is either feeding the BH or converted into stars, we only need to verify the effects of varying the assignment of these stars to the bulge or to the disk component. Performing such a check yields no appreciable variation in our results, even in the DI case. In fact, although the bulge indeed stabilizes the disk (Eqs. (8) and (9)) show that increasing B/T yields lower inflows due to disk instability), the contribution of starbursts to the stellar budget of the model galaxies is minor in the IT case (as shown by Lamastra et al. 2013a) and even more in the DI model, as also supported by observational data (see Rodighiero et al. 2011), except at very high redshifts $z>4$ where the IT mode (not depending on the partition of stars in bulge or disk) is dominant anyway.

The above considerations imply that the growth of the bulge cannot effectively act as a suppression factor to provide lowluminosity AGN in galaxies with massive $\mathrm{BHs}$, which are not produced in the DI scenario (see Fig. 7). In our cosmological framework, these objects can be naturally yielded in the IT scenario, since massive $\mathrm{BHs}$ reside (on average) in high-mass galaxies which a) at low redshift accrete mainly small clumps; and b) have formed from condensations collapsed in biased, highdensity regions of the density field and thus have converted most of their gas into stars (and in the BH feeding) at high redshift, leaving little fuel for the subsequent star formation and $\mathrm{BH}$ feeding processes. The triggering interactions correspond to minor merging (smooth accretion of small lumps, with masses as low 
as $10^{-2}-10^{-3}$ the host mass) and fly-by events. It is not easy to observationally catch one of these events in the act, since (a) they produce intrinsically less disturbance in the host galaxy making them hard to identify morphologically; and (b) the systems only appear disturbed for a short period of time (compared with the AGN active phase, see Storchi-Bergmann et al. 2001). In fact, they are not expected to yield an appreciable enhancement in the clustering of AGN hosts (see Taniguchi 1999; see also Stewart 2009; Lotz et al. 2011). Thus the controversial observational results concerning the clustering of the AGN hosts see Li et al. (2006), Pierce et al. (2007), Darg et al. (2010) for negative evidence, and Alonso et al. (2007), Woods \& Geller (2007), Rogers et al. (2009), Koss (2010), and Ellison (2011) for an enhanced fraction of close pairs of AGN hosts compared to control samples - can hardly provide a definite clue to the minor merging/fly-by scenario (also because of the dependence of the results on the clustering scale). A support for the interactionbased picture can be found in the bluer colours of galaxies close to AGN hosts (see Shirasaki et al. 2011; Kollatschny et al. 2012), and in the observations of specific individual cases (see, e.g., Gonzalez Delgado et al. 2002; Anderson et al. 2013 and references therein). Finally, we note that an alternative (or complementary) possibility is the direct accretion of hot halo gas discussed and implemented in Croton et al. (2006).

As for the relation between inflow and star formation, we recall that an equilibrium amongst the two is assumed in HQ11 model to derive the limit accretion rates attainable in the nucleus. What would happen if this equilibrium were not respected? Actually, different scenarios are possible. First of all, consider the case of a huge mass inflow into the central region of the galaxy: if the gas flows rapidly, it is possible that star formation is not able to contrast the inflow so equilibrium is not reached. Since the surface density of star formation rate $\dot{\Sigma}_{*}$ decreases with increasing radius (Eqs. (A.15), (A.19) and (B.11)), we expect that only the very inner region $\left(R<R_{\mathrm{BH}}\right)$ could suffer from this kind of problem. Furthermore, since the star formation rate is no longer able to contrast the inflow, we can assume that $\dot{\Sigma}_{*}$ could reach a maximum and constant value. Thus, the mass inflow can be simply evaluated using Eq. (5) with $\Sigma_{\mathrm{g}} \simeq$ const instead of using the equilibrium equation (at least in the very inner region). If we use Eq. (5) to evaluate the mass inflow, we obtain a mass inflow $\dot{M} \propto R^{\frac{3}{2}}$, which is actually steeper than that obtained when equilibrium is reached $\left(\dot{M} \propto R^{\frac{5}{6}}\right)$. Since the mass inflow is the same for both cases out of a certain radius, when moving towards the centre the inflow will drop more if the $\dot{M}_{*}$ saturates leading to a less effective final inflow rate onto the central $\mathrm{BH}$. Thus, assuming that equilibrium is always reached provides upper limits to the real mass inflow rate, so our basic conclusions remain valid. On the other hand, it is also possible that when gas density is low enough, both mass inflow and/or star formation could be altered. But since low $\Sigma_{\mathrm{g}}$ is in general correlated with low mass inflow (Eq. (5)), star formation is usually more effective in these cases and consumes most of the gas, suppressing the mass inflow; again, considering the equilibrium, we are actually overestimating the mass inflow rate.

A final fundamental question that remains open concerns the balance between the IT and the DI modes for low-intermediate luminosity AGN. In fact, in such a luminosity range, Fig. 4 shows that both modes could provide the bulk of the AGN population. Although disentangling the contributions from the two is not an easy task, the results in the present paper pave the way to several interesting possibilities. One specific prediction that will allow to constrain the contribution DIs to the
$\mathrm{BH}$ growth is constituted by the luminosity distribution of hosts with high Eddington ratio (see Fig. 8). Other predictions concern the small variance in the paths followed by galaxies evolving in the $M_{\mathrm{BH}}-M_{*}$ plane if $\mathrm{BH}$ are fed by DIs. This means that in such a scenario BH growth $\dot{M}_{\mathrm{BH}}$ and star formation $\dot{M}_{*}$ are tightly related, a situation that can be readily tested by comparing the model predictions with observed $L_{\mathrm{AGN}}-\dot{M}_{*}$ relations or with the observed colour distribution of AGN hosts. We plan to develop such comparisons in detail in our next paper.

Acknowledgements. This work was supported by ASI/INAF contracts I/024/05/0 and I/009/10/0 and PRIN INAF 2011.

\section{References}

Abazajian, K. N., Adelman-McCarthy, J. K., Agueros, M. A., et al. 2009, ApJS, 182,543

Adelman-McCarthy, J. K., Jennifer, K., Agüeron, M. A., et al. 2006, ApJS, 162, 38

Aird, J., Nandra, K., Laird, E.S., et al. 2010, MNRAS, 401, 2531

Aller, M. C., \& Richstone, D. O. 2007, ApJ, 665, 120

Alonso, M. S., Lambas, D. G., Tissera, P. B., \& Coldwell, G. 2007, MNRAS, 375,1017

Anglés-Alcázar, D., Özel, F., \& Davé, R. 2013, ApJ, 770, 5

Anderson, C. S., Johnston, H. M., \& Hunstead, R. W. 2013, MNRAS, 431, 3269 Athanassoula, E. 1992, MNRAS, 259, 328

Bahcall, J. N., Kirhakos, S., Saxe, D. H., \& Schneider, D. P. 1997, ApJ, 479, 642 Baldry, I. K., Glazebrook, K., Brinkmann, J., et al. 2004, ApJ, 600, 681

Baldry, I. K., Driver, S. P., Loveday, J., et al. 2012, MNRAS, 421, 621

Bardeen, J. M., Bond, J. R., Kaiser, N., \& Szalay, A. S. 1986, ApJ, 304, 15 Barnes, J. E., \& Hernquist, L. E. 1991, ApJ, 370, L65

Baugh, C. M. 2006, Rep. Prog. Phys. 69, 3101

Bennert, N., Canalizo, G., Jungwiert, B., et al. 2008, ApJ, 677, 846

Benson, A. J., \& Devereux, N. 2010, MNRAS, 402, 2321

Binney, J., Tremaine, S. 1987, Galactic dynamics (Princeton: Princeton Univ. Press), 747

Bond, J. R., Cole, S., Efstathiou, G., \& Kaiser, N. 1991, ApJ, 379, 440

Bongiorno, A., Zamorani, G., Gavignaud, I., et al. 2007, A\&A, 472, 443

Bouché, N., Cresci, G., Davies, R., et al. 2007, ApJ, 671, 303

Bournaud, F., Combes, F., \& Semelin, B. 2005, MNRAS, 364, L18

Bournaud, F., Elmegreen, B. G., \& Elmegreen, D. M. 2007, ApJ, 670, 237

Bournaud, F., Dekel, A., Teyssier, R., et al. 2011, ApJ, 741, L33

Bournaud, F., Juneau, S., Le Floc'h, E., et al. 2012, ApJ, 757, 81

Bouwens, R. G., Illingworth, G. D., Franx, M., \& Ford, H. 2007, ApJ, 670, 928

Bouwens, R. J., Illingworth, G. D., Oesch, P. A, et al. 2011, ApJ, 737, 90

Bower, R. G. 1991, MNRAS, 248, 332

Bower, R. G., Benson, A. J., Malbon, R., et al. 2006, MNRAS, 370, 645

Brusa, M., Civano, F., Comastri, A., et al. 2010, ApJ, 716, 348

Bruzual, G., Charlot, S. 2003, MNRAS, 344, 1000

Cappellari, M., Bacon, R., Bureau, M., et al. 2006, MNRAS, 366, 1126

Cavaliere, A., \& Vittorini, V. 2000, ApJ, 543, 599

Ceverino, D., Bournaud, F., \& Dekel, A. 2010, MNRAS, 404, 2151

Cirasuolo, M., McLure, R. J., Dunlop, J. S., et al. 2010, MNRAS, 401, 1166

Cisternas, M., Jahnke, K., Inskip, K. J., et al. 2011, ApJ, 726, 57

Civano, F., Brusa, M., Comastri, A., et al. 2011 ApJ, 741, 91

Cole, S., Lacey, C. G., Baugh, C. M., \& Frenk, C. S. 2000, MNRAS, 319, 168

Combes, F., \& Gerin, M. 1985, A\&A, 150, 327

Combes, F., Baker, A. J., Schinnerer, E., et al. 2009, A\&A, 503, 73

Croton, D. J., Springel, V., White, S. D. M., et al. 2006, MNRAS, 365, 11

Cox, T. J., Dutta, S. N., Di Matteo, et al. 2006, ApJ, 650, 791

Cox, T. J., Jonsson, P., Somerville, R. S., Primack, J. R., \& Dekel, A. 2008 , MNRAS, 384, 386

Croom, S. M., Richards, G. T., Shanks, T., et al. 2009, MNRAS, 399, 1755

Daddi, E., Elbaz, D., Walter, F., et al. 2010, ApJ, 714, L118

Darg D. W., Kaviraj, S., Lintott, C. J., et al. 2010, MNRAS, 401, 1552

Davies, R. I.,SaĄnchez, F. M.,Genzel, R., et al. 2007, ApJ, 671, 1388

De Lucia, G., Fontanot, F., Wilman, D., \& Monaco, P. 2011, MNRAS, 414, 1439

de Vries, W. H., Morganti, R., Rottgering, H. J. A., et al. 2002, AJ, 123, 1784

Disney, M. J., Boyce, P. J., Blades, J. C., et al. 1995, Nature, 376, 150

Dotti, M., Ruszkowski, M., Paredi, L., et al. 2009, MNRAS, 396, 1640

Drory, N., Bender, R., Feulner, G., et al. 2004, ApJ, 608, 742

Ebrero, J., Carrera, F. J., Page, M. J., et al. 2009, A\&A, 493, 55

Efstathiou, G., Lake, G., \& Negroponte, J. 1982, MNRAS, 199, 1069 
Eisenhardt, P. R., Stern, D., Brodwin, M., et al. 2004, ApJS, 154, 48 Eliche-Moral, M. C., Gonzalez-Garcia, A. C., Balcells, M., et al. 2008, in Formation and Evolution of Galaxy Disks, eds. J. G. Funes, \& E. M. Corsini (San Francisco, CA: ASP), ASP Conf. Ser. 396, 359

Ellison, S. L., Patton, D. R., Mendel, J. T., \& Scudder, J. M. 2011, MNRAS, 418, 2043

Fanidakis, N., Baugh, C. M., Benson, A. J., et al. 2012, MNRAS, 419, 2797

Ferrarese, L., \& Merritt, D. 2000, ApJ, 539, L9

Fiore, F., O’Dwyer, B., Del Zanna, G., et al. 2012, A\&A, 537, 22

Fisher, D. B., Drory, N. 2011, ApJ, 733, L47

Fontana, A., Salimbeni, S., Grazian, A., et al. 2006, A\&A, 459, 745

Fontanot, F., Cristiani, S., Monaco, P., et al. 2007, A\&A, 461, 39

Friedli, D., \& Martinet, L. 1993, A\&A, 277, 27

Gebhardt, K., Bender, R., Bower, G., et al. 2000, ApJ, 539, L13

Genzel, R., Burkert, A., Bouché, N., et al. 2008, ApJ, 687, 59

Genzel, R., Tacconi, L. J., Gracia-Carpio, J., et al. 2010, MNRAS, 407, 2091

Giavalisco, M., Dickinson, M., Ferguson, H. C., et al. 2004, ApJ, 600, L103

Glikman, E., Djorgovski, S. G., Stern, D., et al. 2011, ApJ, 728, L26

Giallongo, E., Menci, N., Fiore, F., et al. 2012, ApJ, 755, 124

Goldreich, P., \& Lynden-Bell, D. 1965, MNRAS, 130, 97

Goldreich, P., \& Tremaine, S. 1980, ApJ, 241, 425

Gonzalez Delgado, R. M., Arribas, S., Perez, E., \& Heckman, T. 2002, ApJ, 579, 188

Grogin, N. A., Conselice, C. J., Chatzichristou, E., et al. 2005, ApJ 627, L97

Häring, N., \& Rix, H.-W. 2004, ApJ, 604, L89

Heller, C. H., \& Shlosman, I. 1994, ApJ, 424, 84

Hennawi, J. F., Myers, A. D., Shen, Y., et al. 2010, ApJ, 719, 1672

Henriques, B. M. B., \& Thomas, P. A. 2010, MNRAS, 403, 768

Hernquist, L. 1989, Nature, 340, 687

Hernquist, L., \& Mihos, J. C. 1995, ApJ, 448, 41

Hickox, R. C., Jones, C., Forman, W. R., et al. 2009, ApJ, 696, 891

Hicks, E. K. S., Davies R. I., Malkan M. A., et al. 2009, ApJ, 696, 448

Hildebrandt, H., Pielorz, J., Erben, T., et al. 2009, A\&A, 498, 725

Hirschmann, M., Somerville, R. S., Naab, T., \& Burkert, A. 2012, MNRAS, 426, 237

Hopkins, P. F., \& Quataert, E. 2010, MNRAS, 407, 1529

Hopkins, P. F., \& Quataert, E. 2011, MNRAS, 411, 1027 (HQ11)

Hopkins, P. F., Richards, G. T., \& Hernquist, L. 2007, ApJ, 654, 731

Hopkins, P. H., Somerville, R. S., Cox, T. J., et al. 2009, MNRAS, 397, 802

Hutchings, J. B. 1987, ApJ, 320, 122

Jiang, L., Fan, X., Bian, F. et al. 2009, AJ, 138, 305

Jogee, S. 2006, Lect. Notes Phys, 693, 143

Jones, D. H., Peterson, B. A., Colless, M., \& Saunders, W. 2006, MNRAS, 396, 535

Kaspi, S., Smith, P. S., Netzer, H., et al. 2000, ApJ, 533, 631

Kauffmann, G., \& Heckman, T. M. 2009, MNRAS, 397, 135

Kauffmann, G., Heckman, T. M., Tremonti, C., et al. 2003, MNRAS, 346, 1055

Kenter, A., Murray, S. S., Forman, W. R., et al. 2005, ApJS, 161, 9

Khochfar, S., \& Silk, J. 2006, MNRAS, 370, 902

King, I. 1962, AJ, 67, 471

Kirhakos, S., Bahcall, J. N., Schneider, D. P., Kristian, J. 1999, ApJ, 520, 67

Kocevski, D. D., Faber, S. M., Mozena, M., et al. 2012, ApJ, 744, 148

Kollatschny, W., Reichstein, A., \& Zetzl, M. 2012, A\&A, 548, 37

Kollmeier, J. A., Oken, C. A., Kochanek, C. S., et al. 2006, ApJ, 648, 128

Kormendy, J., \& Richstone, D. 1995, ARA\&A, 33, 581

Koss, M., Mushotzky, R., Veilleux, S., \& Winter, L. 2010, ApJ, 716, L125

Lacey, C., \& Cole, S. 1993, MNRAS, 262, 627

La Franca, F., Fiore, F., Comastri, A., et al. 2005, ApJ, 635, 864

Lamastra, A., Menci, N., Maiolino, R. Fiore, F., \& Merloni, A. 2010, MNRAS, 405, 29

Lamastra, A., Menci, N., Fiore, F., \& Santini, P. 2013a, A\&A, 552, 44

Lamastra, A., Menci, N., Fiore, F., et al. 2013b, A\&A, 559, A56

Li, C., \& White, S. D. M. 2009, MNRAS, 398, 2177

Li, C., Kauffmann, G., Wang, L., et al. 2006, MNRAS, 373, 457

Lilly, S. J., Le Fevre, O., Renzini, A., et al. 2007, ApJS, 172, 70

Lin, C. C., Yuan, C., \& Shu, F. H. 1969, ApJ, 155, 721

Lotz, J. M., Jonsson, P., Cox, T. J., et al.

Lynden-Bell, D., \& Kalnajs, A. J. 1972, MNRAS, 157, 1

Maciejewski, W., \& Sparke, L. S. 2000, MNRAS, 313, 745

Mayer, L., Kazantzidis, S., Madau, P., et al. 2007, Science, 316, 1874

Magorrian, J., Tremaine, S., Richstone, D., et al. 1998, AJ, 115, 2285

Makino, J., \& Hut, P. 1997, ApJ, 481, 83
Marconi, A., \& Hunt, L. K. 2003, ApJ, 589, L21

Marconi, A., Risaliti, G., Gilli, R., et al. 2004, MNRAS, 351, 169

McGurk, R. C., Max, C. E., Rosario, D. J., et al. 2011, ApJ, 738, 2

Menci, N., Cavaliere, A., Fontana, A., et al. 2003, ApJ, 587, L63

Menci, N., Cavaliere, A., Fontana, A., Giallongo, E., \& Poli, F. 2004, ApJ, 604, 12

Menci, N., Fontana, A., Giallongo, E., Grazian, A., \& Salimbeni, S. 2006, ApJ, 647, 753

Menci, N., Fiore, F., Puccetti, S., \& Cavaliere, A. 2008, ApJ, 686, 219

Mo, H. J, Mao, S., \& White, S. D. M. 1998, MNRAS, 295, 319

Murray, S. S., Kenter, A., Forman, W. R., et al. 2005, ApJS, 161, 1

Navarro, J. F., Frenk, C. S., \& White, S. D. M. 1997, ApJ, 490, 493

Netzer, H. 2009a, MNRAS, 399, 1907

Netzer, H. 2009b, ApJ, 695, 793

Ostriker, E. C., Shu, F. H., \& Adams, F. C. 1992, ApJ, 399, 192

Panter, B., Jimenez, R., Heavens, A. F., \& Charlot, S. 2007, MNRAS, 378, 1150

Parry, O. H., Eke, V. R., \& Frenk, C. S. 2009, MNRAS, 396, 1972

Pierce, C. M., Lotz, J. M., Laird, E. S., et al. 2007, ApJ, 660, L19

Reddy, N. A., \& Steidel, C. S. 2009, ApJ, 692, 778

Richards, G. T., Strauss, M. A., Fan, X., et al. 2006, ApJ, 131, 2766

Robertson, B., Cox, T. J., Hernquist, L., et al. 2006a, ApJ, 641, 21

Robertson, B., Hernquist, L., Cox, T. J., et al. 2006b, ApJ, 641, 90

Rodighiero, G., Daddi, E., Baronchelli, I., et al. 2011, ApJ, 739, L40

Rogers, B., Ferreras, I., Kaviraj, S., Pasquali, A., \& Sarzi, M. 2009, MNRAS, 399, 2172

Rosario, D. J., Mozena, M., Wuyts, S., et al. 2013a, 763, 59

Rosario, D. J., Trakhtenbrot, B., Lutz, D., et al. 2013b, A\&A, 560, A72

Salucci, P., Szuszkiewicz, E., Monaco, P., \& Danese, L. 1999, MNRAS, 307, 637

Sanders, D. B., \& Mirabel, I. F. 1996, ARA\&A, 34, 749

Santini, P., Maiolino, R., Magnelli, B., et al. 2014, A\&A, 562, 30

Saslaw, W. C. 1985, Gravitational Physics of Stellar and Galactic Systems (Cambridge: Cambridge Univ. Press)

Schwarz, M. P. 1984, MNRAS, 209, 93

Shankar, F., Weinberg, D. H., \& Miralda-Escude, J. 2009, ApJ, 690, 20

Shankar, F., Marulli, F., Mathur, S., Bernardi, M., \& Bournaud, F. 2012, A\&A, 540, A23

Shankar, F., Weinberg, D. H., \& Miralda-Escude, J. 2013, MNRAS, 428, 421

Shirasaki, Y., Tanaka, M., Ohishi, M., et al. 2011, PASJ, 63, 469

Shlosman, I., Frank, J., \& Begelman, M. C. 1989, Nature, 338, 45

Shu, F. H., Tremaine, S., Adams, F. C., \& Ruden, S. P. 1990, ApJ, 358, 495

Siana, B., Polletta, M., Smith, H. E., et al. 2008, ApJ, 675, 49

Silverman, J. D., Lamareille, F., Maier, C., et al. 2009, ApJ, 696, 396

Silverman, J. D., Kampczyk, P., Jahnke, K., et al. 2011, ApJ, 743, 2

Somerville, R. S., \& Primack, J. R. 1999, MNRAS, 310, 1087

Somerville, R. S., Gilmore, R. C., Primack J. R., \& Dominguez, A. 2012, MNRAS, 423, 1992

Spergel, D. N., Bean, R., Doré, O., et al. 2007, ApJS, 171, 377

Stewart, K. R. 2009, in Galaxy Evolution: Emerging Insights and Future Challenges, eds. S. Jogee, I. Marinova, L. Hao, G. A. Blanc, ASP Conf. Ser. 419, 243

Storchi-Bergmann, T., González Delgado, R. M., Schmitt, H. R., Cid, F. R., \& Heckman, T. 2001, in ASP Conf. Proc. 249, eds. J. H. Knapen, J. E. Beckman, I. Shlosman, \& T. J. Mahoney, 259 (San Francisco: ASP)

Taniguchi, Y. 1999, ApJ, 524, 65

Taylor, J. E., Babul, A. 2001, ApJ, 559, 716

Teyssier, R., Chapon, D., \& Bournaud, F. 2010, ApJ, 720, L149

Toomre, A. 1969, ApJ, 158, 899

Trakhtenbrot, B., \& Netzer, H. 2012, MNRAS, 427, 3081

Treister, E., Schawinski, K., Urry, C. M., \& Simmons, B. D. 2012, ApJ, 758, L39

Tremaine, S., Gebhardt, K., Bender, R., et al. 2002, ApJ, 574, 740

Trump, J. R., Impey, C. D., Elvis, M., et al. 2009, ApJ, 696, 1195

Ueda, Y., et al. 2014, ApJ, in press

van der Burg, R. F. J., Hildebrandt, H., \& Erben, T. 2010, A\&A, 523, 74

Villforth, C., Hamann, F., Rosario, D. J., et al. 2014, MNRAS, 439, 3342

Villar-Martin, M., Tadhunter, C., Humphrey, A., et al. 2011, MNRAS, 416, 262

Villar-Martin, M, Cabrera Lavers, A., Bessiere, P., et al. 2012, MNRAS, 423, 80

Woods, D. F., \& Geller, M. J. 2007, AJ, 134, 527

Yates, M. G., Miller, L., \& Peacock, J. A. 1989, MNRAS, 240, 129

York, D. G., Adelman, J., Anderson, J. E., et al. 2000, AJ, 120, 1579

Younger, J. D., Hopkins, P. F., Cox, T. J., \& Hernquist, L. 2008, ApJ, 686, 815

Yu, Q., \& Tremaine, S. 2002, MNRAS, 335, 965 


\section{Appendix A: Black hole accretion rate and nuclear star formation rate from disk instabilities: the case of power-law surface density profiles}

Following the computation in HQ11, we solve Eqs. (5) and (6) for both $\Sigma_{\mathrm{g}}$ and $\dot{M}_{\text {infl. }}$ To this aim, we consider two distinct regions: an outer region where the potential is dominated by the disk, and an inner region where the $\mathrm{BH}$ gravity is dominant. The radius $R_{\mathrm{BH}} \sim 10 \mathrm{pc}$ marks the separations between the two regions, while the scale $R_{\text {acc }} \sim 0.1 \mathrm{pc}$ corresponds to the inner bound of the innermost regions where we want to compute the $\mathrm{BH}$ accretion rate in Eq. (7).

\section{A.1. The potential}

To estimate the potential in the outer regions (from $\sim 10 \mathrm{pc}$ to $\sim 100 \mathrm{pc}$ ), HQ11 adopt the WKB approximation to estimate the perturbation amplitude $\Phi_{a} \approx|a|_{\max } 2 \pi G \Sigma_{\mathrm{d}} R|k R|^{-1}$, taking $|k R| \sim 1$ since they are considering global modes. On the basis of aimed $N$-body simulations (Hopkins \& Quataert 2010a) the dominant mode is assumed to be $m=2$ and the maximal perturbation amplitude $|a|_{\max } \approx 0.3 f_{\mathrm{d}}(R)$ is taken to be proportional to the disk fraction $f_{\mathrm{d}}(R)$ for any radius $R$. In the inner one (from $R_{\text {acc }} \sim 0.1$ pc to $\left.R_{\mathrm{BH}} \sim 10 \mathrm{pc}\right)$, the potential is dominated by the $\mathrm{BH}$ and $\Phi_{a} \approx|a|_{\max } G M_{\mathrm{d}}\left(R_{\mathrm{BH}}\right) / R_{\mathrm{BH}}$, with $|a|_{\max } \approx 0.2 f_{\mathrm{d}}\left(R_{\mathrm{BH}}\right)$. In this region, the dominant mode is $m=1$, also motivated by simulations.

\section{A.2. The gas surface density}

In both regions, disk, bulge and gas surface densities are assumed to be described by power-law profiles, that is, $\Sigma_{\mathrm{d}} \propto R^{-\eta_{\mathrm{d}}}$, $\Sigma_{\mathrm{b}} \propto R^{-\eta_{\mathrm{d}}}, \Sigma_{\mathrm{g}} \propto R^{-\eta_{\mathrm{g}}}$. In addition, HQ11 assume the disk to be stellar dominated so that the total surface density $\Sigma_{\mathrm{t}}=\Sigma_{b}+\Sigma_{\mathrm{d}} \propto$ $R^{-\eta_{\mathrm{d}}}$, and the disk fraction $f_{\mathrm{d}}=\int \Sigma_{\mathrm{d}} R \mathrm{~d} R / \int \Sigma_{\mathrm{t}} R \mathrm{~d} R$ is constant with radius.

With this assumptions it is possible to solve Eqs. (5) and (6) for both the gas density $\Sigma_{\mathrm{g}}$ and the mass inflow rate $\mathrm{d} M_{\mathrm{infl}} / \mathrm{d} t$. In particular, in the inner region $R_{\mathrm{acc}} \leq R \leq R_{\mathrm{BH}}$ where the $\mathrm{BH}$ dominates the potential $\Phi$ in Eq. (5), Eq. (6) yields

$$
\begin{array}{r}
\left(\frac{\Sigma_{\mathrm{g}}}{\Sigma_{\mathrm{k}}}\right)_{\text {inner }}^{\eta_{\mathrm{k}}-1}=\frac{3 \eta_{\mathrm{k}}-4}{20 \pi\left(\eta_{\mathrm{k}}-1\right)} \sqrt{\frac{G M_{\mathrm{BH}}}{R_{\mathrm{BH}}^{3}}} t_{\mathrm{k}}\left(\frac{R}{R_{\mathrm{BH}}}\right)^{-1 / 2} f_{\mathrm{d}} \\
R_{\mathrm{acc}} \leq R \leq R_{\mathrm{BH}}
\end{array}
$$

while in the outer region $R_{\mathrm{BH}} \leq R \leq R_{0}$, where the potential $\Phi$ is dominated by the disk, the same equations yield

$$
\left(\frac{\Sigma_{\mathrm{g}}}{\Sigma_{\mathrm{k}}}\right)_{\text {outer }}^{\eta_{\mathrm{k}}-1}=\alpha_{\mathrm{d}}\left(\frac{\Sigma_{\mathrm{d}}}{\Sigma_{\mathrm{t}}}\right)^{2} \Omega t_{\mathrm{k}}
$$

with

$\alpha_{\mathrm{d}}=\frac{m a_{0}-2 \eta_{\mathrm{d}}-\eta_{\mathrm{g}}+3\left(\eta_{\mathrm{d}}+1\right) / 2 \mid\left(2-\eta_{\mathrm{d}}\right)^{2}}{8 \pi\left(2-\left(\eta_{\mathrm{d}}+1\right) / 2\right)} \quad R_{\mathrm{BH}} \leq R \leq R_{0}$

where $\Omega=\left[G 2 \pi \int_{0}^{R}\left(\Sigma_{\mathrm{d}}+\Sigma_{b}\right) R \mathrm{~d} R / R^{3}\right]^{1 / 2}$ is the angular velocity of the disk.

\section{A.3. The boundary conditions}

To compute the accretion flow in the inner region $R_{\text {acc }} \leq R \leq$ $R_{\mathrm{BH}}$, we can use Eq. (A.1) to solve Eq. (6). However, the two density profiles in Eqs. (A.1) and (A.2) are not matched to ensure a continuous mass flux at the boundaries $R_{\mathrm{BH}}$ and $R_{0}$. In principle, one could impose a continuity condition for such equations at all times. Since the boundaries move as accretion proceeds, this would require a detailed treatment following the dynamical evolution of the boundaries and of the mass flux across them. Following HQ11, we adopt a simplified approach by we keeping the density profiles in Eqs. (A.1) and (A.2), and we impose the mass conservation directly in Eqs. (6). Thus, we write the latter as

$\frac{\mathrm{d} M_{\mathrm{infl}}}{\mathrm{d} t}=2 \pi R d R \frac{\Sigma_{\mathrm{k}}}{t_{\mathrm{k}}}\left(\frac{\Sigma_{\mathrm{g}}}{\Sigma_{\mathrm{k}}}\right)^{\eta_{\mathrm{k}}} A\left(R_{\mathrm{BH}}, R_{0}\right)$

where $A\left(R_{\mathrm{BH}}, R_{0}\right)$ expresses the proper boundary conditions, i.e., the mass conservation across the boundaries $R_{\mathrm{BH}}$ and $R_{0}$. To compute such a quantity, we note that the mass accreted into the inner region $\left(R_{\mathrm{acc}} \leq R \leq R_{\mathrm{BH}}\right)$ must equal the disk mass that has flown across $R_{\mathrm{BH}}$. We indicate with $\Delta M_{\mathrm{g}}$ the mass inside the boundary $R_{\mathrm{BH}}$ computed after integrating Eq. (A.1), and with $A\left(R_{\mathrm{BH}}, R_{0}\right) \Delta M_{\mathrm{g}}$ the actual mass accreted in the inner region after matching with it with the mass flown from the disk. In a time interval $\Delta t$, we must have

$$
\begin{gathered}
\frac{A\left(R_{\mathrm{BH}}, R_{0}\right) \Delta M_{\mathrm{g}}}{\Delta M_{\mathrm{g}}}=A\left(R_{\mathrm{BH}}\right) \\
=\frac{\dot{M}_{\mathrm{g}}\left(R_{\mathrm{BH}}, \text { outside }\right)}{\dot{M}_{\mathrm{g}}\left(R_{\mathrm{BH}}, \text { inside }\right)+\int_{R_{\mathrm{acc}}}^{R_{\mathrm{BH}}} \Sigma_{\mathrm{g}, \text { inner }}(R) 2 \pi R d R / \Delta t} .
\end{gathered}
$$

We must have $\dot{M}_{\mathrm{g}}\left(R_{\mathrm{BH}}\right.$, outside $)=\dot{M}_{\mathrm{g}}\left(R_{\mathrm{BH}}\right.$, inside $)$. Estimating exactly such a quantity would require a dynamical description of the variation of the boundary with time and of the mass flux across it. However, a simple - though approximate estimate of such a quantity can be obtained by computing it as the mass lost from a disk during $\Delta t$ (divided by $\Delta t$ ). This can be obtained after integrating the disk density profile A. 2 extrapolated down to $R=0$, so that $\dot{M}_{\mathrm{g}}\left(R_{\mathrm{BH}}\right.$, outside $) \approx$ $B\left(R_{0}\right) \int_{0}^{R_{\mathrm{BH}}} \Sigma_{\mathrm{g}, \text { outer }}(R) 2 \pi R \mathrm{~d} R / \Delta t$. The quantity $B\left(R_{0}\right)$ is the normalization corresponding to the boundary condition at the galactic scale $R_{0}$, which ensures that the mass flux outside the boundary $R_{0}$ matches with that in the region $R_{\mathrm{BH}} \leq R \leq R_{0}$. We then obtain

$A\left(R_{\mathrm{BH}}, R_{0}\right)=$

$$
\begin{gathered}
\overline{1+\int_{R_{\mathrm{acc}}}^{R_{\mathrm{BH}}} \Sigma_{\mathrm{g}, \text { inner }}(R) 2 \pi R d R / B\left(R_{0}\right) \int_{R_{\mathrm{acc}}}^{R_{\mathrm{BH}}} \Sigma_{\mathrm{g}, \text { outer }}(R) 2 \pi R \mathrm{~d} R} \\
=\frac{1}{1+2.5 f_{\mathrm{d}}^{-4 / 3} / B\left(R_{0}\right)}
\end{gathered}
$$

where the last equality follows after performing the integrals over $\Sigma_{\mathrm{g}, \text { inner }}$ and $\Sigma_{\mathrm{g}, \text { outer }}$ given in Eqs. (A.1) and (A.2). The quantity $A\left(R_{\mathrm{BH}}, R_{0}\right)$ acts like a suppression factor; when the mass inflow obtained using the (unmatched) density distributions (A.1) and (A.2) yields an inflow rate that exceeds the gas mass actually available from the disk at the position $R_{\mathrm{BH}}$, it suppresses the mass flux so as to maintain it lower or equal to this mass. In fact, for high values of $\Delta M_{\mathrm{g}}$ (outside, $\left.R_{\mathrm{BH}}\right)$ we get $A\left(R_{\mathrm{BH}}, R_{0}\right)=1$. No suppression is needed since the disk mass available from the disk is high. 
The quantity $B\left(R_{0}\right)$ in Eq. (A.3) can be obtained by iterating the same mass-matching procedure exactly, but applying it to the outer boundary $R_{0}$. In terms of the disk mass $M_{\mathrm{d}}\left(R_{0}\right)$ within $R_{0}$ and of the corresponding gas mass $M_{\mathrm{g}}\left(R_{0}\right)=f_{\mathrm{gas}} M_{\mathrm{d}}\left(R_{0}\right)$, the result reads as

$$
B\left(R_{0}\right)=\frac{1}{1+\int_{R_{\text {acc }}}^{R_{0}} \Sigma_{\mathrm{g}, \text { outer }}(R) 2 \pi R \mathrm{~d} R / M_{\mathrm{g}}\left(R_{0}\right)}=\frac{1}{1+f_{0} / f_{\text {gas }}}
$$

with

$$
f_{0} \approx 0.2 f_{\mathrm{d}}^{2}\left(\frac{M_{\mathrm{d}}\left(R_{0}\right)}{10^{9} M_{\odot}}\right)^{-1 / 3} \quad f_{\text {gas }} \equiv \frac{M_{\mathrm{g}}\left(\mathrm{R}_{0}\right)}{M_{\mathrm{d}}\left(R_{0}\right)} .
$$

The physical meaning of the boundary factor $B\left(R_{0}\right)$ is to match the gas available in the galactic disk (given by the semi-analytic model) with the gas corresponding to the profile $\Sigma_{\mathrm{g}}$ inside $R_{0}$. In the original HQ11 model, the gas density profile $\Sigma_{\mathrm{g}}$ in the external region has a slightly higher normalization to interpolate between the marginal and the strong orbit crossing regimes; however, when such a factor is included, its effects on Eqs. (A.6) and (A.5) balance, leaving our results unchanged.

\section{A.4. The $B H$ inflow rate}

We now proceed to solve Eq. (A.3) to derive the mass inflow rate $M_{\text {infl }}$ close to the inner bound $R_{\text {acc. HQ1 }}$ first consider Eq. (6) in a ring close to $R_{\text {acc }}$ and small enough that the surface density $\Sigma_{\mathrm{g}}$ can be considered to be constant. This yields

$$
\frac{\mathrm{d} M_{\mathrm{infl}}}{\mathrm{d} t}\left(R_{\mathrm{acc}}\right) \simeq \pi R_{\mathrm{acc}}^{2} \frac{\Sigma_{\mathrm{k}}}{t_{\mathrm{k}}}\left(\frac{\Sigma_{\mathrm{g}}\left(R_{\mathrm{acc}}\right)}{\Sigma_{\mathrm{k}}}\right)^{\eta_{\mathrm{k}}} A\left(R_{\mathrm{BH}}, R_{0}\right) .
$$

After substituting Eq. (A.1) computed ad the inner radius $R_{\text {acc }}$ the above equation reads

$$
\begin{aligned}
\frac{\mathrm{d} M_{\mathrm{BH}}}{\mathrm{d} t} \equiv & \frac{\mathrm{d} M_{\mathrm{infl}}}{\mathrm{d} t}\left(R_{\mathrm{acc}}\right)=\pi \Sigma_{\mathrm{k}} t_{\mathrm{k}}^{\frac{1}{\eta_{\mathrm{k}}-1}}\left(\frac{R_{\mathrm{acc}}}{R_{\mathrm{BH}}}\right)^{\frac{3 \eta_{\mathrm{k}}-4}{2\left(\eta_{\mathrm{k}}-1\right)}} \\
& \times R_{\mathrm{BH}}^{2}\left[\frac{3 \eta_{\mathrm{k}}-4}{20 \pi\left(\eta_{\mathrm{k}}-1\right)}\left(\frac{G M_{\mathrm{BH}}}{R_{\mathrm{BH}}^{3}}\right)^{\frac{1}{2}} f_{\mathrm{d}}\right]^{\frac{\eta_{\mathrm{k}}}{\eta_{\mathrm{k}}-1}} A\left(R_{\mathrm{BH}}, R_{0}\right) .
\end{aligned}
$$

Equation (A.8) represents the mass inflow in the inner region corresponding to the $\mathrm{BH}$ accretion rate. We can relate $R_{\mathrm{BH}}$ to $M_{\mathrm{BH}}$ in terms of the disk mass within $R_{\mathrm{BH}}$ through the relation $M_{\mathrm{t}}\left(R_{\mathrm{BH}}\right) \simeq M_{\mathrm{BH}}$. The mass $M_{\mathrm{t}}$ is computed as $M_{\mathrm{t}}\left(R_{\mathrm{BH}}\right)=$ $\int_{0}^{R_{\mathrm{BH}}} 2 \pi \Sigma_{\mathrm{t}} R \mathrm{~d} R$. For a power-law profile $\Sigma_{\mathrm{t}}=A_{\Sigma_{\mathrm{t}}} R^{-\eta_{\mathrm{d}}}$ we can readily express $R_{\mathrm{BH}}$ as a function of $M_{\mathrm{t}}=M_{\mathrm{BH}}$ to obtain

$R_{\mathrm{BH}}=\left(M_{\mathrm{BH}} \frac{2-\eta_{\mathrm{d}}}{2 \pi A_{\Sigma_{\mathrm{t}}}}\right)^{\frac{1}{2-\eta_{\mathrm{d}}}}$.

Substituting in Eq. (A.8) yields

$$
\begin{aligned}
\frac{\mathrm{d} M_{\mathrm{BH}}}{\mathrm{d} t}= & \pi \Sigma_{\mathrm{k}} t_{\mathrm{k}}^{\frac{1}{\eta_{\mathrm{k}}-1}}\left[\frac{3 \eta_{\mathrm{k}}-4}{20 \pi\left(\eta_{\mathrm{k}}-1\right)} G^{\frac{1}{2}}\right]^{\frac{\eta_{\mathrm{k}}}{\eta_{\mathrm{k}}-1}}\left(\frac{R_{\mathrm{acc}}}{R_{\mathrm{BH}}}\right)^{\frac{3 \eta_{\mathrm{k}}-4}{2\left(\eta_{\mathrm{k}}-1\right)}} \\
& \times M_{\mathrm{BH}}^{\frac{3 \eta_{\mathrm{k}}-4-\eta_{\mathrm{k}} \eta_{\mathrm{d}}}{2\left(\eta_{\mathrm{k}}-1\right)\left(2-\eta_{\mathrm{d}}\right)}} f_{\mathrm{d}}^{\frac{\eta_{\mathrm{k}}}{\eta_{\mathrm{k}}-1}}\left(\frac{2-\eta_{\mathrm{d}}}{2 \pi A_{\Sigma_{\mathrm{t}}}}\right)^{\frac{\eta_{\mathrm{k}}-4}{2\left(\eta_{\mathrm{k}}-1\right)\left(2-\eta_{\mathrm{d}}\right)}} A\left(R_{\mathrm{BH}}, R_{0}\right) .
\end{aligned}
$$

We are now interested in expressing Eq. (A.8) using physical quantities evaluated far from the very central region, at galactic scales $R=R_{0} \sim 10^{2}-10^{3} \mathrm{pc}$, in order to derive the $\mathrm{BH}$ accretion rate from quantities computed from our semi-analytic galaxy formation model. To this aim, we note that, for any disk profile $\Sigma_{\mathrm{d}}$, it is possible to combine $R_{0}$ and the associated enclosed disk mass $M_{\mathrm{d}}\left(R_{0}\right)=\int_{0}^{R_{0}} 2 \pi \Sigma_{\mathrm{d}} R \mathrm{~d} R$ to yield convenient constant quantities. In particular for a power-law profile, after some algebra, the last two factors in Eq. (A.10) can be recast as

$$
f_{\mathrm{d}}^{\frac{\eta_{\mathrm{k}}}{\eta_{\mathrm{k}}-1}}\left(\frac{2-\eta_{\mathrm{d}}}{2 \pi A_{\Sigma_{\mathrm{t}}}}\right)^{\frac{\eta_{\mathrm{k}}-4}{2\left(\eta_{\mathrm{k}}-1\right)\left(2-\eta_{\mathrm{d}}\right)}}=M_{\mathrm{d}}\left(R_{0}\right)^{\frac{4-\eta_{\mathrm{k}}}{2\left(\eta_{\mathrm{k}}-1\right)\left(2-\eta_{\mathrm{d}}\right)}} R_{0}^{\frac{\eta_{\mathrm{k}}-4}{2\left(\eta_{\mathrm{k}}-1\right)}} f_{\mathrm{d}}^{\frac{5 \eta_{\mathrm{k}}-4-2 \eta_{\mathrm{d}} \eta_{\mathrm{k}}}{2\left(\eta_{\mathrm{k}}-1\right)\left(2-\eta_{\mathrm{d}}\right)}} .
$$

After substituting in Eq. (A.10) this reads as

$$
\begin{aligned}
& \frac{\mathrm{d} M_{\mathrm{BH}}}{\mathrm{d} t}=\pi \Sigma_{\mathrm{k}} t_{\mathrm{k}}^{\frac{1}{\eta_{\mathrm{k}}-1}}\left[\frac{3 \eta_{\mathrm{k}}-4}{20 \pi\left(\eta_{\mathrm{k}}-1\right)} G^{\frac{1}{2}}\right]^{\frac{\eta_{\mathrm{k}}}{\eta_{\mathrm{k}}-1}}\left(\frac{R_{\mathrm{acc}}}{R_{\mathrm{BH}}}\right)^{\frac{3 \eta_{\mathrm{k}}-4}{2\left(\eta_{\mathrm{k}}-1\right)}} \\
& \quad \times M_{\mathrm{BH}}^{\frac{3 \eta_{\mathrm{k}}-4-\eta_{\mathrm{k}} \eta_{\mathrm{d}}}{2\left(\eta_{\mathrm{k}}-1\right)\left(2-\eta_{\mathrm{d}}\right)}} M_{\mathrm{d}}\left(R_{0}\right)^{\frac{4-\eta_{\mathrm{k}}}{2\left(\eta_{\mathrm{k}}-1\right)\left(2-\eta_{\mathrm{d}}\right)}} R_{0}^{\frac{\eta_{\mathrm{k}}-4}{2\left(\eta_{\mathrm{k}}-1\right)}} f_{\mathrm{d}}^{\frac{5 \eta_{\mathrm{k}}-4-2 \eta_{\mathrm{d}} \eta_{\mathrm{k}}}{2\left(\eta_{\mathrm{k}}-1\right)\left(2-\eta_{\mathrm{d}}\right)}} A\left(R_{\mathrm{BH}}, R_{0}\right) .
\end{aligned}
$$

We can express the above equation in physical units, which yields

$$
\begin{aligned}
& \frac{\mathrm{d} M_{\mathrm{BH}}}{\mathrm{d} t}=\alpha\left(\eta_{\mathrm{k}}, \eta_{\mathrm{d}}\right)\left(\frac{R_{\mathrm{acc}}}{10^{-2} R_{\mathrm{BH}}}\right)^{\frac{3 \eta_{\mathrm{k}}-4}{2\left(\eta_{\mathrm{k}}-1\right)}}\left(\frac{M_{\mathrm{BH}}}{10^{8} M_{\odot}}\right)^{\frac{3 \eta_{\mathrm{k}}-4-\eta_{\mathrm{k}} \eta_{\mathrm{d}}}{2\left(\eta_{\mathrm{k}}-1\right)\left(2-\eta_{\mathrm{d}}\right)}} \\
& \times\left(\frac{M_{\mathrm{d}}\left(R_{0}\right)}{10^{9} M_{\odot}}\right)^{\frac{4-\eta_{\mathrm{k}}}{2\left(\eta_{\mathrm{k}}-1\right)\left(2-\eta_{\mathrm{d}}\right)}}\left(\frac{R_{0}}{100 \mathrm{pc}}\right)^{\frac{\eta_{\mathrm{k}}-4}{2\left(\eta_{\mathrm{k}}-1\right)}} f_{\mathrm{d}}^{\frac{5 \eta_{\mathrm{k}}-4-2 \eta_{\mathrm{d}} \eta_{\mathrm{k}}}{2\left(\eta_{\mathrm{k}}-1\right)\left(2-\eta_{\mathrm{d}}\right)}} A\left(R_{\mathrm{BH}}, R_{0}\right) \frac{M_{\odot}}{\mathrm{yr}},
\end{aligned}
$$

where we have gathered all constant terms into the normalization

$\alpha\left(\eta_{\mathrm{k}}, \eta_{\mathrm{d}}\right)=\pi(0.4)^{\frac{1}{\eta_{\mathrm{k}}-1}}\left(2.2 \frac{3 \eta_{\mathrm{k}}-4}{20 \pi\left(\eta_{\mathrm{k}}-1\right)}\right)^{\frac{\eta_{\mathrm{k}}}{\eta_{\mathrm{k}}-1}} 10^{\frac{32-15 \eta_{\mathrm{k}}-14 \eta_{\mathrm{d}}+7 \eta_{\mathrm{k}} \eta_{\mathrm{d}}}{2\left(\eta_{\mathrm{k}}-1\right)\left(2-\eta_{\mathrm{d}}\right)}}$

The above expression is the $\mathrm{BH}$ accretion rate for generic powerlaw surface density profiles. After assuming a power law index $\eta_{\mathrm{d}}=1 / 2$ for the gas surface density profile and an in$\operatorname{dex} \eta_{\mathrm{k}}=7 / 4$ for the Kennicut-Schmidt star formation law, the above equation yields Eq. (9) in the main text and a normalization $\alpha\left(\eta_{\mathrm{k}}, \eta_{\mathrm{d}}\right)=0.18$. As noted by HQ11, assuming different values for $\eta_{\mathrm{d}}$ and $\eta_{\mathrm{k}}$ has only a minor effect on the exponents in Eq. (A.13), but has a significant impact on $\alpha\left(\eta_{\mathrm{k}}, \eta_{\mathrm{d}}\right)$. For example, changing the $\eta_{\mathrm{k}}$ to the value $\eta_{\mathrm{k}}=3 / 2$ yields $\alpha\left(\eta_{\mathrm{k}}, \eta_{\mathrm{d}}\right)=3.17$. In the main text, we assume a reference value for $\alpha\left(\eta_{\mathrm{k}}, \eta_{\mathrm{d}}\right)=10$ to investigate the maximal contribution of the DI mode to the evolution of the AGN population.

\section{A.5. The nuclear star formation rate}

The contribution to the star formation rate can be calculated integrating star formation surface density rate $\dot{\Sigma}_{*}$ over the region of interest. The latter is related to the gas surface density by the Schmidt-Kennicut law $\Sigma_{*} \propto\left(\Sigma_{\mathrm{g}}\right)^{\eta_{\mathrm{k}}}$. For the inner region, $\Sigma_{\mathrm{g}}$ is described by Eq. (A.1). Since all the terms in Eq. (A.1) are constant but the radius, we obtain

$\frac{\mathrm{d} \Sigma_{*}}{\mathrm{~d} t} \propto R^{-\frac{\eta_{\mathrm{k}}}{2\left(\eta_{\mathrm{k}}-1\right)}}$. 
The normalization can be estimated considering the value that the star formation rate assumes in $R=R_{\text {acc }}$. This can be done easily since the mass inflow in $R=R_{\text {acc }}$ is strictly related to star formation surface density rate by Eq. (6) and gives $\dot{M}_{\mathrm{BH}}=$ $\dot{M}_{\text {infl }}\left(R_{\text {acc }}\right) \simeq \pi R_{\text {acc }}^{2} \dot{\Sigma}_{*}\left(R_{\text {acc }}\right)$. Thus, the star formation surface density rate in the inner region is equal to

$\frac{\mathrm{d} \Sigma_{*}}{\mathrm{~d} t}=\frac{\mathrm{d} M_{\mathrm{BH}}}{\mathrm{d} t} \frac{1}{\pi R_{\mathrm{acc}}^{2}}\left(\frac{R}{R_{\mathrm{acc}}}\right)^{-\frac{\eta_{\mathrm{k}}}{2\left(\eta_{\mathrm{k}}-1\right)}}$.

The contribution to the star formation rate in the inner region is then obtained by integrating (A.16) from $R_{\mathrm{acc}}$ to $R_{\mathrm{BH}}$ :

$$
\begin{aligned}
\frac{\mathrm{d} M_{*, \text { inner }}}{\mathrm{d} t} & =\int_{R_{\mathrm{acc}}}^{R_{\mathrm{BH}}} 2 \pi \frac{\mathrm{d} M_{\mathrm{BH}}}{\mathrm{d} t} \frac{1}{\pi R_{\mathrm{acc}}^{2}}\left(\frac{R}{R_{\mathrm{acc}}}\right)^{-\frac{\eta_{\mathrm{k}}}{2\left(\eta_{\mathrm{k}}-1\right)}} R \mathrm{~d} R \\
& =\frac{\mathrm{d} M_{\mathrm{BH}}}{\mathrm{d} t} \frac{4\left(\eta_{\mathrm{k}}-1\right)}{3 \eta_{\mathrm{k}}-4} R_{\mathrm{acc}}^{\frac{-3 \eta_{\mathrm{k}}+4}{2\left(\eta_{\mathrm{k}}-1\right)}}\left(R_{\mathrm{BH}}^{\frac{3 \eta_{\mathrm{k}}-4}{2\left(\eta_{\mathrm{k}}-1\right)}}-R_{\mathrm{acc}}^{\frac{3 \eta_{\mathrm{k}}-4}{2\left(\eta_{\mathrm{k}}-1\right)}}\right) .
\end{aligned}
$$

For the fiducial model, assuming $\eta_{\mathrm{k}}=7 / 4$ and $R_{\mathrm{acc}} \simeq 10^{-2} R_{\mathrm{BH}}$, we obtain

$$
\frac{\mathrm{d} M_{*, \text { inner }}}{\mathrm{d} t} \simeq 109 \frac{\mathrm{d} M_{\mathrm{BH}}}{d t} \text {. }
$$

The same calculations can be performed for the outer region using the corresponding $\Sigma_{\mathrm{g}} \propto R^{-\frac{\eta_{\mathrm{d}}+1}{2}}$; here the scaling and the exponent $\alpha_{\mathrm{d}}$ are given in Eq. (A.2). This yields

$\frac{\mathrm{d} \Sigma_{*}}{\mathrm{~d} t} \propto R^{-\frac{\eta_{\eta\left(\eta_{\mathrm{d}}+1\right)}}{2\left(\eta_{\mathrm{k}}-1\right)}}$

The normalization can be computed imposing continuity between the inner and the outer region. Using Eq. (A.16)

$$
\frac{\mathrm{d} \Sigma_{*}}{\mathrm{~d} t}=\frac{\mathrm{d} M_{\mathrm{BH}}}{\mathrm{d} t} \frac{1}{\pi R_{\mathrm{acc}}^{2}}\left(\frac{R_{\mathrm{BH}}}{R_{\mathrm{acc}}}\right)^{-\frac{\eta_{\mathrm{k}}}{2\left(\eta_{\mathrm{k}}-1\right)}}\left(\frac{R}{R_{\mathrm{BH}}}\right)^{-\frac{\eta_{\mathrm{k}}\left(\eta_{\mathrm{d}}+1\right)}{2\left(\eta_{\mathrm{k}}-1\right)}} .
$$

Integrating Eq. (A.20) from $R_{\mathrm{BH}}$ to $R_{0}$, we obtain the star formation rate in the outer region for the fiducial model:

$$
\begin{aligned}
\frac{\mathrm{d} M_{*, \text { outer }}}{\mathrm{d} t}= & \frac{\mathrm{d} M_{\mathrm{BH}}}{\mathrm{d} t} \frac{4\left(\eta_{\mathrm{k}}-1\right)}{\left(-\eta_{\mathrm{k}} \eta_{\mathrm{d}}+3 \eta_{\mathrm{k}}-4\right) R_{\mathrm{acc}}^{2}}\left(\frac{R_{\mathrm{BH}}}{R_{\mathrm{acc}}}\right)^{-\frac{\eta_{\mathrm{k}}}{2\left(\eta_{\mathrm{k}}-1\right)}} \\
& \times R_{\mathrm{BH}}^{\frac{\eta_{\mathrm{k}}\left(\eta_{\mathrm{d}}+1\right)}{2\left(\eta_{\mathrm{k}}-1\right)}}\left(R_{0}^{\frac{-\eta_{\mathrm{k}} \eta_{\mathrm{d}}+3 \eta_{\mathrm{k}}-4}{2\left(\eta_{\mathrm{k}}-1\right)}}-R_{\mathrm{BH}}^{\frac{-\eta_{\mathrm{k}} \eta_{\mathrm{d}}+3 \eta_{\mathrm{k}}-4}{2\left(\eta_{\mathrm{k}}-1\right)}}\right)
\end{aligned}
$$

Assuming $\eta_{\mathrm{k}}=7 / 4, \eta_{\mathrm{d}}=1 / 2$, and $R_{\mathrm{acc}} \simeq 10^{-2} R_{\mathrm{BH}}$, we obtain

$$
\frac{\mathrm{d} M_{*, \text { outer }}}{\mathrm{d} t} \simeq 289 \frac{\mathrm{d} M_{\mathrm{BH}}}{\mathrm{d} t} \text {. }
$$

\section{Appendix B: Black hole accretion rate and nuclear star formation rate from disk instabilities: the case of exponential surface density profiles}

Here we follow the same procedure as adopted in Appendix A, changing only the surface density profile (and the corresponding potential) of the galactic disk. In particular, we consider a thin disk whose density profile is described by the following exponential-law:

$$
\Sigma_{\mathrm{d}}(R)=\Sigma_{0} \mathrm{e}^{-R / R_{\mathrm{d}}}
$$

where $R_{\mathrm{d}}$ is the exponential length of the disk and its order a few $\mathrm{kpc}$. The potential associated to the disk is (Binney \& Tremaine 1987)

$$
\Phi(R)=-\pi G \Sigma_{0} R\left[I_{0}(y) K_{1}(y)-I_{1}(y) K_{0}(y)\right]
$$

where $y \equiv R / 2 R_{\mathrm{d}}$ and $I_{n}, K_{n}$ are the modified Bessel function of first and second kinds. Since both the disk and the bulge exponential length are much greater than scales of our interest, we can use the asymptotic form for the modified Bessel function; as a result, we obtain

$$
\begin{aligned}
& \Phi(R) \approx-2 \pi G \Sigma_{0} R_{\mathrm{d}} \\
& \Sigma_{\mathrm{d}}(R) \approx \Sigma_{0} .
\end{aligned}
$$

We note that in this case the disk surface density and the potential are computed self-consistently, at variance with the previous case where we assumed a WKB form for the potential and a power-law form for the surface density.

Following the same procedure as adopted in Appendix A, we consider two regions: an outer region where the potential is dominated by the disk, and an inner region where the $\mathrm{BH}$ gravity is dominant. The radius $R_{\mathrm{BH}} \sim 10 \mathrm{pc}$ marks the separations between the two regions, while the scale $R_{\text {acc }} \sim 0.1$ pc corresponds to the inner bound of the innermost regions where we want to compute the $\mathrm{BH}$ accretion rate. The potential of the disk is only needed to calculate the analytic expression for $\Sigma_{\mathrm{g}}$ in the outer region, where the $\mathrm{BH}$ does not dominate the potential.

In the inner region, the potential is dominated by the $\mathrm{BH}$, and the computation of the $\mathrm{BH}$ accretion rate follows the lines shown in Appendix A exactly, changing only the scaling of the disk radial profile by adopting $\eta_{\mathrm{d}}=0$, to achieve a constant $\Sigma_{\mathrm{d}}$ as given by Eq. (B.4). Thus, the $\mathrm{BH}$ accretion rate is given by the same Eq. (A.13) with $\eta_{\mathrm{d}}=0$. However, the boundary condition $A\left(R_{\mathrm{BH}}, R_{0}\right)$ is different from Appendix $\mathrm{A}$, since it depends on the gas surface density $\Sigma_{\mathrm{g} \text {,outer }}$ in the region $R>R_{\mathrm{BH}}$ where the potential and the surface densities need to be recomputed (see Eqs. (A.5) and (A.6)). Also, the contribution to the nuclear star formation from such a region $R>R_{\mathrm{BH}}$ has to be recomputed, since it also depends on $\Sigma_{\mathrm{g}, \text { outer }}$.

To compute $\Sigma_{\mathrm{g}, \text { outer }}$ in the outer region we start from the perturbed potential in the outer region

$\left|\Phi_{a}\right| \approx a_{0} f_{\mathrm{d}} \Phi_{0}=\frac{2 a_{0} \pi^{2} G^{2} \Sigma_{0}^{2} R_{\mathrm{d}}}{\Omega^{2} R}$

with $a_{0} \sim 0.3$ and $m=2$, motivated by simulations. Substituting the above expression into Eq. (5) we obtain for the outer region (from $R_{\mathrm{BH}}$ to $R_{0}$ )

$$
\begin{aligned}
\frac{\partial}{\partial R}\left(\frac{\mathrm{d} M_{\mathrm{infl}}}{\mathrm{d} t}\right) & =m a_{0} \Sigma_{\mathrm{g}} \frac{1}{\Omega^{3} R^{2}} \frac{\pi^{2} G^{2} \Sigma_{0}^{2} R_{\mathrm{d}}}{\left(2+v_{\Omega}\right)}\left|-\eta_{\mathrm{g}}-3 v_{\Omega}-1\right| \\
& =\alpha_{E} \pi \Omega\left(\frac{\Sigma_{0}}{\Sigma_{\mathrm{t}}}\right)^{2} \Sigma_{\mathrm{g}} R_{\mathrm{d}}
\end{aligned}
$$

with

$v_{u} \equiv \frac{\partial \ln u}{\partial \ln R} \quad$ and $\quad \alpha_{E} \equiv \frac{m a_{0}}{\pi} \frac{\left|-\eta_{\mathrm{g}}-3 v_{\Omega}-1\right|}{\left(2+v_{\Omega}\right)}$

where we have used the relation $\Omega^{2} R=G \int 2 \pi \Sigma_{\mathrm{t}} R \mathrm{~d} R$, and $\Sigma_{\mathrm{t}}$ is the total (disk + bulge) surface density profiles (see Appendix A). Substituting such an expression in Eq. (6) yields

$\left(\frac{\Sigma_{\mathrm{g}}}{\Sigma_{\mathrm{k}}}\right)_{\text {outer }}^{\eta_{\mathrm{k}}-1}=\frac{\alpha_{E}}{2}\left(\frac{\Sigma_{0}}{\Sigma_{\mathrm{t}}}\right)^{2} \frac{\Omega t_{\mathrm{k}} R_{\mathrm{d}}}{R}$.

That is the equation for $\Sigma_{\mathrm{g}}$ in the outer region $R_{\mathrm{BH}} \leq R \leq R_{0}$ and the counterpart of Eq. (A.2) in the case of an exponential disk surface density profile; in the inner region $R_{\mathrm{acc}} \leq R \leq R_{\mathrm{BH}}$, the analytic expression for $\Sigma_{\mathrm{g}}$ is the same as Eq. (A.1), since this 
region is dominated by $\mathrm{BH}$ potential. We can now recompute the boundary conditions $A\left(R_{\mathrm{BH}}, R_{0}\right)$ and $B\left(R_{0}\right)$. To this aim, we use Eqs. (A.5) and (A.6), but adopting the expression in Eq. (B.8) for the gas surface density in the outer region $\Sigma_{\mathrm{g} \text {,outer }}$. This yields

$A\left(R_{\mathrm{BH}}, R_{0}\right)=\left(1+\frac{4 \eta_{\mathrm{k}}-7-\eta_{\mathrm{d}}}{4 \eta_{\mathrm{k}}-5}\left(\frac{\Sigma_{0}}{\Sigma_{\mathrm{t}}} \frac{R_{\mathrm{d}}}{R_{\mathrm{BH}}} \frac{\alpha_{E}}{2 \alpha_{\mathrm{BH}}}\right)^{-\frac{1}{\eta_{\mathrm{k}}-1}} / B\left(R_{0}\right)\right)^{-1}$.

In practice, the right-hand term in the parenthesis is extremely small, owing to the choice of $\eta_{\mathrm{k}}, \eta_{\mathrm{d}}$ ( $\eta_{\mathrm{d}} \sim 0$ since $\Sigma_{0}$ is almost constant) and to the factor $R_{\mathrm{d}} / R_{\mathrm{BH}}$, so that the above expression for the case of an exponential disk always yields $A\left(R_{\mathrm{BH}}, R_{0}\right) \approx 1$, independently of the outer boundary condition, which anyway reads (in the case $\eta_{\mathrm{k}}=7 / 4$ )

$B\left(R_{0}\right) \approx\left[1+26 \frac{f_{0}}{f_{\text {gas }}}\left(\frac{R_{\mathrm{d}}}{R_{0}}\right)^{4 / 3}\right]^{-1}$

where $f_{0}$ and $f_{\text {gas }}$ are those given in Eq. (A.6). Thus, in the main text, we adopt the excellent approximation $A\left(R_{\mathrm{BH}}, R_{0}\right)=1$.

As for the nuclear star formation, the contribution from the inner region $R_{\mathrm{acc}} \leq R \leq R_{\mathrm{BH}}$ is the same as given in Eq. (A.17), since in this region the potential is dominated by the BH. In the outer region, we follow the procedure presented in Sect. A5, based on the relation $\Sigma_{* \text {,outer }} \propto \Sigma_{\mathrm{g}, \text { outer }}^{\eta_{\mathrm{k}}}$, with the latter quantity taken from Eq. (B.8). This yields

$\frac{\mathrm{d} \Sigma_{*}}{\mathrm{~d} t}=\frac{\mathrm{d} M_{\mathrm{BH}}}{\mathrm{d} t} \frac{1}{\pi R_{\mathrm{acc}}^{2}}\left(\frac{R_{\mathrm{BH}}}{R_{\mathrm{acc}}}\right)^{-\frac{\eta_{\mathrm{k}}}{2\left(\eta_{\mathrm{k}}-1\right)}}\left(\frac{R}{R_{\mathrm{BH}}}\right)^{-\frac{\eta_{\mathrm{k}}\left(\eta_{\mathrm{d}}+3\right)}{2\left(\eta_{\mathrm{k}}-1\right)}}$.

Integrating Eq. (B.11) from $R_{\mathrm{BH}}$ to $R_{0}$, we obtain

$$
\begin{aligned}
\frac{\mathrm{d} M_{*, \text { outer }}}{\mathrm{d} t}= & \frac{\mathrm{d} M_{\mathrm{BH}}}{\mathrm{d} t} \frac{4\left(\eta_{\mathrm{k}}-1\right)}{\left(-\eta_{\mathrm{k}} \eta_{\mathrm{d}}+\eta_{\mathrm{k}}-4\right) R_{\mathrm{acc}}^{2}}\left(\frac{R_{\mathrm{BH}}}{R_{\mathrm{acc}}}\right)^{-\frac{\eta_{\mathrm{k}}}{2\left(\eta_{\mathrm{k}}-1\right)}} \\
& \times R_{\mathrm{BH}}^{\frac{\eta_{\mathrm{k}}\left(\eta_{\mathrm{d}}+3\right)}{2\left(\eta_{\mathrm{k}}-1\right)}}\left(R_{0}^{\frac{-\eta_{\mathrm{k}} \eta_{\mathrm{d}}+\eta_{\mathrm{k}}-4}{2\left(\eta_{\mathrm{k}}-1\right)}}-R_{\mathrm{BH}}^{\frac{-\eta_{\mathrm{k}} \eta_{\mathrm{d}}+\eta_{\mathrm{k}}-4}{2\left(\eta_{\mathrm{k}}-1\right)}}\right)
\end{aligned}
$$

Taking $\eta_{\mathrm{k}}=7 / 4, \eta_{\mathrm{d}}=0$, and $R_{0}=100 \mathrm{pc}$,

$\frac{\mathrm{d} M_{*, \text { outer,exp }}}{\mathrm{d} t}=\frac{4}{3} \frac{\mathrm{d} M_{\mathrm{BH}}}{\mathrm{d} t} R_{\mathrm{BH}}^{\frac{7}{3}} R_{\mathrm{acc}}^{-\frac{5}{6}}\left(R_{\mathrm{BH}}^{-\frac{3}{2}}-R_{0}^{-\frac{3}{2}}\right) \simeq 60 \frac{\mathrm{d} M_{\mathrm{BH}}}{\mathrm{d} t}$.

At variance with the case of power law profile given in Eq. (A.21), the star formation rate does not diverge with increasing $R_{0}$, due to the exponential decay of the disk surface density. 